\title{
Long-term plasticity of inhibitory synapses in the hippocampus and spatial learning depends on matrix metalloproteinase 3
}

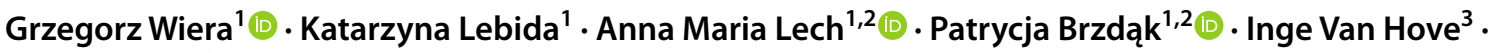 \\ Lies De Groef ${ }^{3,4} \cdot$ Lieve Moons $^{3,4} \cdot$ Enrica Maria Petrini ${ }^{5} \cdot$ Andrea Barberis $^{5} \cdot$ Jerzy W. Mozrzymas $^{1}$
}

Received: 27 April 2020 / Revised: 19 August 2020 / Accepted: 3 September 2020 / Published online: 21 September 2020

(c) The Author(s) 2020

\begin{abstract}
Learning and memory are known to depend on synaptic plasticity. Whereas the involvement of plastic changes at excitatory synapses is well established, plasticity mechanisms at inhibitory synapses only start to be discovered. Extracellular proteolysis is known to be a key factor in glutamatergic plasticity but nothing is known about its role at GABAergic synapses. We reveal that pharmacological inhibition of MMP3 activity or genetic knockout of the Mmp3 gene abolishes induction of postsynaptic iLTP. Moreover, the application of exogenous active MMP3 mimics major iLTP manifestations: increased mIPSCs amplitude, enlargement of synaptic gephyrin clusters, and a decrease in the diffusion coefficient of synaptic $\mathrm{GABA}_{\mathrm{A}}$ receptors that favors their entrapment within the synapse. Finally, we found that MMP3 deficient mice show faster spatial learning in Morris water maze and enhanced contextual fear conditioning. We conclude that MMP3 plays a key role in iLTP mechanisms and in the behaviors that presumably in part depend on GABAergic plasticity.
\end{abstract}

Keywords GABA $\cdot$ iLTP $\cdot$ Mmps $\cdot$ Metalloproteinases $\cdot$ Synaptic plasticity $\cdot$ Synaptic inhibition

Grzegorz Wiera and Katarzyna Lebida equal contribution.

Electronic supplementary material The online version of this article (https://doi.org/10.1007/s00018-020-03640-6) contains supplementary material, which is available to authorized users.

Grzegorz Wiera

grzegorz.wiera@umed.wroc.pl

$\triangle$ Katarzyna Lebida

katarzyna.lebida@umed.wroc.pl

1 Laboratory of Neuroscience, Department of Biophysics, Wroclaw Medical University, 50-367, Wroclaw, Poland

2 Laboratory of Cellular Neurobiology, Department of Physiology and Molecular Neurobiology, Wroclaw University, 50-205, Wroclaw, Poland

3 Neural Circuit Development and Regeneration Research Group, Department of Biology, University of Leuven (KU Leuven), 3000 Leuven, Belgium

4 Leuven Brain Institute (LBI), KU Leuven, Leuven, Belgium

5 Laboratory of Synaptic Plasticity of Inhibitory Networks, Fondazione Istituto Italiano Di Tecnologia, 16163 Genoa, Italy

\section{Introduction}

Learning and memory formation have been primarily associated with the plasticity of excitatory glutamatergic synapses [1], the mechanisms of which have been studied for more than four decades [2,3]. More recently, however, inhibitory GABAergic synapses were found to exhibit many forms of long-term plasticity that is putatively important in learning and memory $[4,5]$. At the circuit level, plastic changes at GABAergic synapses are thought to regulate numerous phenomena, such as the plasticity of excitatory transmission [6], timing of the auditory critical period [7], stabilization of neuronal dynamics that prevents overexcitation [8], as well as establishment, size and reciprocal interference of engrams $[9,10]$. Nonetheless, a comprehensive molecular description of GABAergic plasticity has been limited by the diversity of inhibitory neurons and complexity of already known multifarious types of GABAergic plasticity that can be induced by homo- or heterosynaptic mechanisms and expressed pre- or postsynaptically.

A large body of evidence shows that the extracellular proteolysis plays a crucial role in the plasticity of glutamatergic excitatory synapses $[11,12]$. For example, through the cleavage of identified proteins, matrix metalloproteinase 9 
(MMP9) regulates the consolidation of long-term potentiation (LTP) [13], the structural plasticity of dendritic spines $[14,15]$ or synaptic plasticity that is related to addiction [16, 17] and stress-induced social impairments [18]. Moreover, another metalloproteinase-MMP3 - through the cleavage of unknown substrates, has been shown to be necessary for cross-modal plasticity in the visual cortex [19], the L-type channel-dependent component of LTP [20], and the LTP of $N$-methyl-D-aspartate (NMDA) receptor transmission in the hippocampus [21]. MMP3 has been also implicated in mediating habituation-induced plasticity in the hippocampus and prefrontal cortex [22] and inhibition of this protease was suggested to alter long-term plasticity and prevent learning in the Morris water maze task [23]. However, as noted by the authors of these studies, the inhibitors that were used were not entirely specific for MMP3. Additionally, learning in the passive avoidance conditioning paradigm is associated with higher MMP3 expression in the hippocampus [24]. However, in contrast to the well-established role of extracellular proteolysis in excitatory plasticity and learning, little is known about its role in GABAergic plasticity and related cognitive functions.

The present study investigated the role of extracellular MMPs in the mechanisms of GABAergic inhibitory LTP (iLTP) in the hippocampus. We utilized a model of GABAergic iLTP, in which plasticity is induced heterosynaptically and characterized by the postsynaptic locus of expression. This form of plasticity has been described in several brain regions, including the hippocampus [25], cortex [26], and cerebellum [27]. Using electrophysiological recordings, morphological analyses of synapses, and single-particle tracking approaches, we found that the activity of MMP3 is critical for the expression of NMDA-induced iLTP and the immobilization of synaptic $\mathrm{GABA}_{\mathrm{A}}$ receptors. Moreover, exogenous MMP3 activity increased amplitude of miniature inhibitory synaptic currents (mIPSCs) and the size of gephyrin synaptic clusters. Finally, we evaluated spatial learning in MMP3 deficient $\left(\mathrm{Mmp3}^{--}\right)$mice, which exhibited faster learning in the Morris water maze and an enhancement of contextual fear conditioning. Overall, these findings reveal that the extracellular proteolytic activity of MMP3 regulates GABAergic iLTP and shapes spatial learning.

\section{Results}

\section{Induction of iLTP at CA1 inhibitory synapses depends on extracellular proteolytic activity}

Under the present experimental conditions, $\mathrm{GABA}_{\mathrm{A}}$ receptor-mediated miniature inhibitory postsynaptic current (mIPSC) amplitudes that were measured in slices from CA1 hippocampal pyramidal neurons in the whole-cell configuration were stable for at least $60 \mathrm{~min}$. The mean amplitude at a holding voltage of $-70 \mathrm{mV}$ was $31.46 \pm 3.62$ $\mathrm{pA}$, and the frequency was $2.18 \pm 0.2 \mathrm{~Hz}$. To induce postsynaptic iLTP, we adopted a chemical protocol [25] and applied NMDA $(20 \mu \mathrm{M})$ in the bath solution for $3 \mathrm{~min}$. As a result of NMDA treatment, a stable mIPSCs potentiation was observed (Fig. 1a), reaching mean amplitude enhancement of $1.25 \pm 0.05$ fold (relative to baseline). As explained in the "Methods" section, the extent of iLTP was assessed 20-22 min after plasticity induction and calculated relative to mIPSC amplitude values before NMDA administration ( $p=0.003$; paired $t$ test; Fig. 1b). As expected [25], no changes in the relative mean frequency of mIPSCs were noticed $(1.00 \pm 0.08, p=1$; Supplementary Fig. 1a). To evaluate the involvement of extracellular proteolysis in this form of plasticity, we first used FN-439 $(180 \mu \mathrm{M})$, a broad-spectrum MMPs inhibitor. Interestingly, in the presence of FN-439, iLTP was impaired $(0.98 \pm 0.05, p=0.002$; Fig. 1a-c). The representative raw and average traces that are shown in Fig. 1d demonstrate that NMDA stimulation increased the mIPSC amplitude under control conditions, but not when MMPs were blocked by FN-439.

In the next series of experiments, we sought to identify the type of MMP that is involved in GABAergic plasticity in our model using more specific MMPs inhibitors. Interestingly, bath application of UK-356618, which inhibits MMP3, MMP9, and MMP13, not only prevented the induction of iLTP but led also to an iLTP-to-iLTD conversion (Ctrl DMSO: 1.16 \pm 0.05;UK-356618: $0.94 \pm 0.03$; $p=0.002$; Fig. $1 \mathrm{e}-\mathrm{g})$. On the contrary, the MMP2/9 inhibitor SB-3CT $(10 \mu \mathrm{M})$ had no effect on iLTP $(1.18 \pm 0.05$; Ctrl DMSO: $1.17 \pm 0.08 ; p=0.534$; Supplementary Fig. 1b, c). Altogether, this pharmacological approach provided evidence that MMP3 or MMP13 activity is required for hippocampal chemical iLTP.

\section{Mmp3 knockout prevents iLTP}

Since the inhibitors that we used had limited selectivity for individual MMPs, the subsequent experiments evaluated iLTP in $M m p 3^{-1-}$ mice [28]. We first compared the basal amplitude, frequency, rise time, and mean decay time constant of mIPSCs in slices from the $\mathrm{Mmp}^{-/-}$group compared with those of the wild-type (WT). Deficiency of the Mmp3 gene did not affect mIPSC amplitude (WT: $39.07 \pm 2.54 \mathrm{pA} ; \mathrm{Mmp3}^{-/-}: 42.60 \pm 2.83 \mathrm{pA} ; p=0.433$; Supplementary Fig. 2a), frequency (WT: $2.42 \pm 0.01 \mathrm{~Hz}$; $\mathrm{Mmp3}^{-/-}: 2.32 \pm 0.098 \mathrm{~Hz} ; p=0.676$; Supplementary

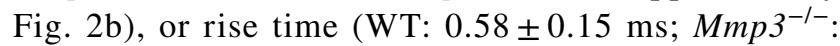
$0.56 \pm 0.02 \mathrm{~ms} ; p=0.568$; Supplementary Fig. $2 \mathrm{c}$ ), but prolonged mIPSC decay kinetics (WT: $15.54 \pm 0.52 \mathrm{~ms}$; 

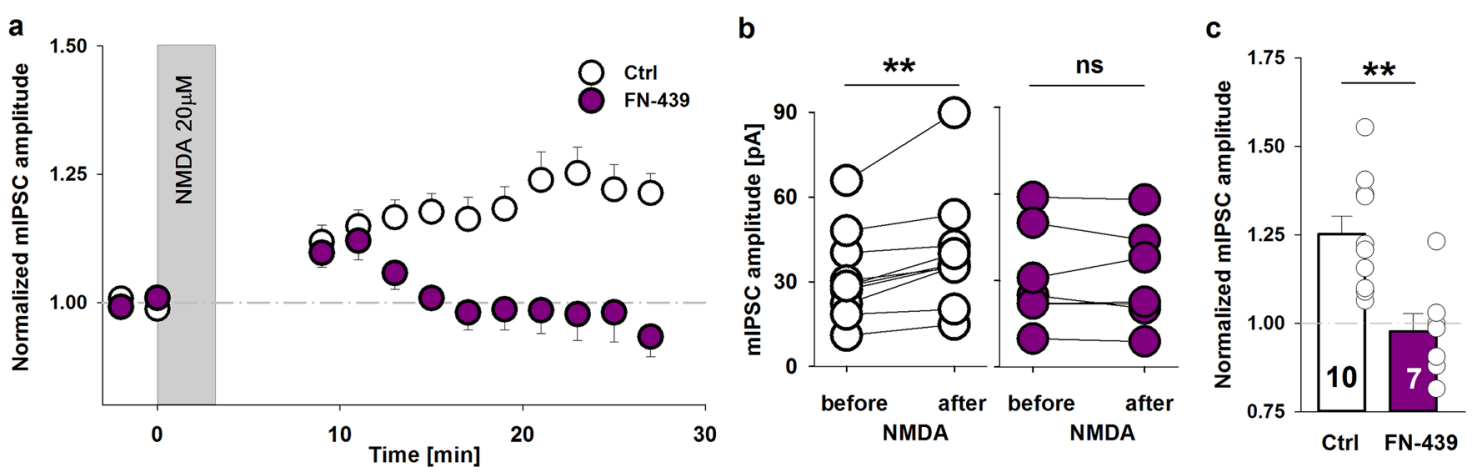

d

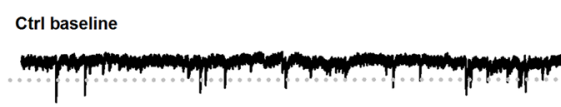

Ctrl after NMDA
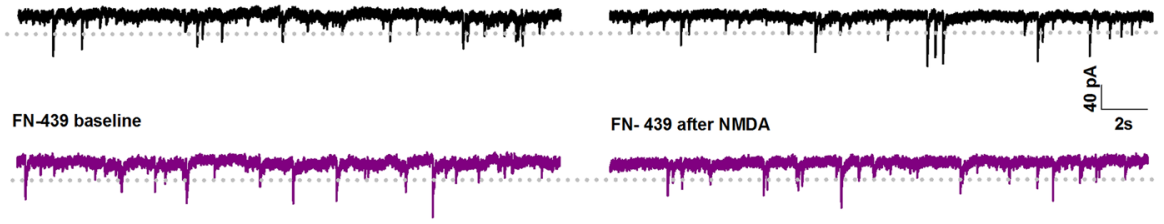

FN- 439 after NMDA
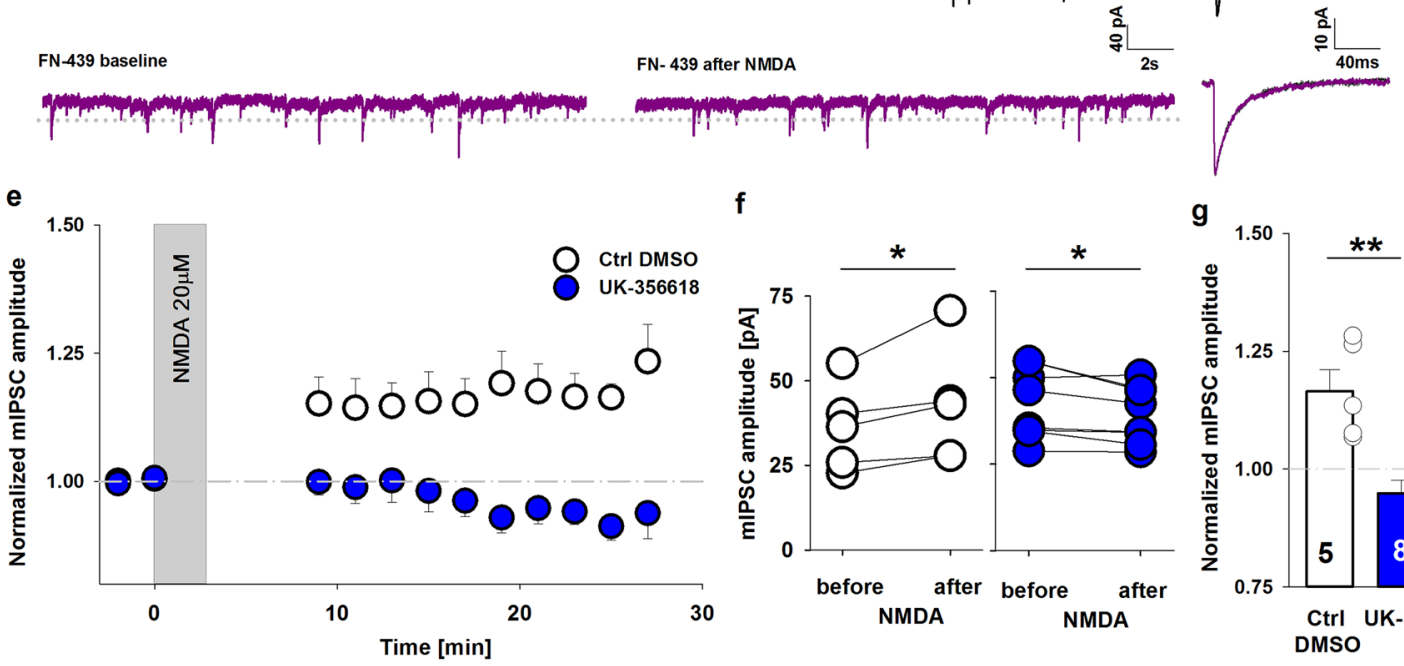

f

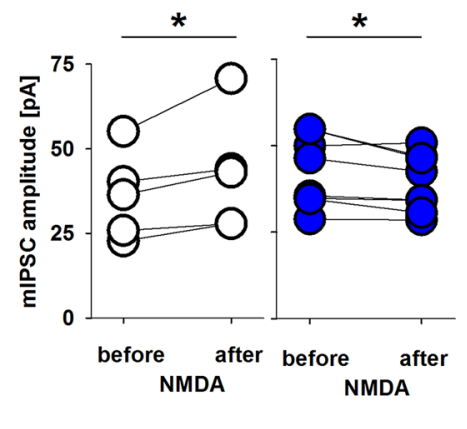

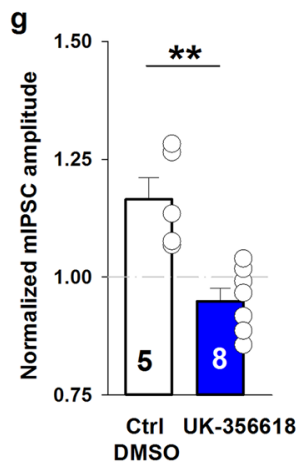

h
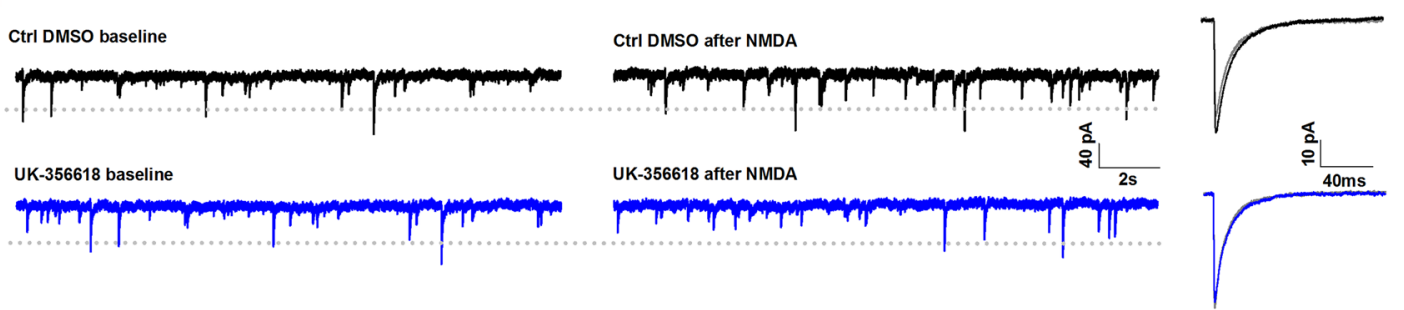

Fig. 1 Matrix metalloproteinases are involved in GABAergic plasticity in the hippocampus. a Application of NMDA ( $3 \mathrm{~min}, 20 \mu \mathrm{M}$, gray bar) induced iLTP in CA1 hippocampal pyramidal cells under control conditions (white) but not in FN-439-treated slices (magenta). b Significant increase in mIPSC amplitude recorded from a single pyramidal cell after NMDA treatment under control conditions (paired $t$ test) and the lack of this effect in the presence of FN-439. c Statistics for iLTP magnitude (measured 20-22 min after iLTP induction) in control (white) and FN-439-treated slices (magenta, unpaired $t$ test). d Representative recordings and averaged traces that show the influence of NMDA application on mIPSC amplitude and frequency in control slices (top) and FN-439-treated slices (bottom). On the right side grey lines show superimposed traces before NMDA stimulation while black and magenta line represents traces after iLTP induction (in control and FN-439 treated group respectively). e Time course of relative mIPSC amplitude after iLTP induction, recorded from control slices (white) and in the presence of UK-356618 (blue). f Summary plot of mIPSC amplitudes that were recorded from control slices (white) and UK-356618-treated slices (blue) before and after NMDA application (paired $t$ test). $\mathbf{g}$ Statistics of the effect of UK-356618 on the size of iLTP measured 20-22 min after NMDA stimulation (Mann-Whitney $U$ test). $\mathbf{h}$ Sample traces of mIPSC recordings from an individual pyramidal cell (on the left) and averaged traces (on the right) under control conditions (top) and in the presence of UK-356618 (bottom), before and after the bath application of NMDA. In the case of averaged currents the gray lines correspond to the MIPSC recorded before the NMDA stimulation while the black and blue colors of lines represent the mIPSC measured after NMDA treatment. In $b$ and $f$, the average mIPSC amplitude of each neuron is shown as one circle. The numbers on the bars refer to the number of recordings. In $\mathrm{d}$ and $\mathrm{h}$ dotted line indicates the mean amplitude of baseline mIPSC. $* p<0.05$, $* * p<0.01 . n s$ nonsignificant 
$\mathrm{Mmp}^{-/-}: 18.14 \pm 0.76 \mathrm{~ms} ; p=0.006$; Supplementary Fig. 2d).

We next investigated the effects of MMP3 deletion on iLTP that was evoked by brief NMDA stimulation. In the $\mathrm{Mmp}^{-/-}$group, no iLTP was induced (relative mIPSC amplitude enhancement: $1.03 \pm 0.03 ; p=0.95$; Fig. $2 \mathrm{a}-\mathrm{c}$ ), in contrast to the respective wild-type control $(1.25 \pm 0.05$; $p=0.005$; Fig. 2a-c); as also shown in Fig. 2d presenting raw and averaged exemplary traces. Interestingly, in wildtype slices, successful iLTP induction did not affect the mIPSC rise time (relative rise time: $1.05 \pm 0.04 ; p=0.21$; Supplementary Fig. 3a) but prolonged the mean decay time constant (relative $\tau_{\text {mean }}: 1.25 \pm 0.06 ; p=0.006$; Supplementary Fig. 3b). However, in $\mathrm{Mmp}^{-/-}$slices, neither the mIPSC decay phase nor rise time was affected by iLTP induction (relative $\tau_{\text {mean }}: 1.05 \pm 0.04, p=0.2$; Supplementary Fig. 3a; relative rise time: $1.01 \pm 0.04, p=0.94$; Supplementary Fig. 3b). Thus, these findings are further supporting the conclusion that the absence of MMP3 activity impaired iLTP.

a

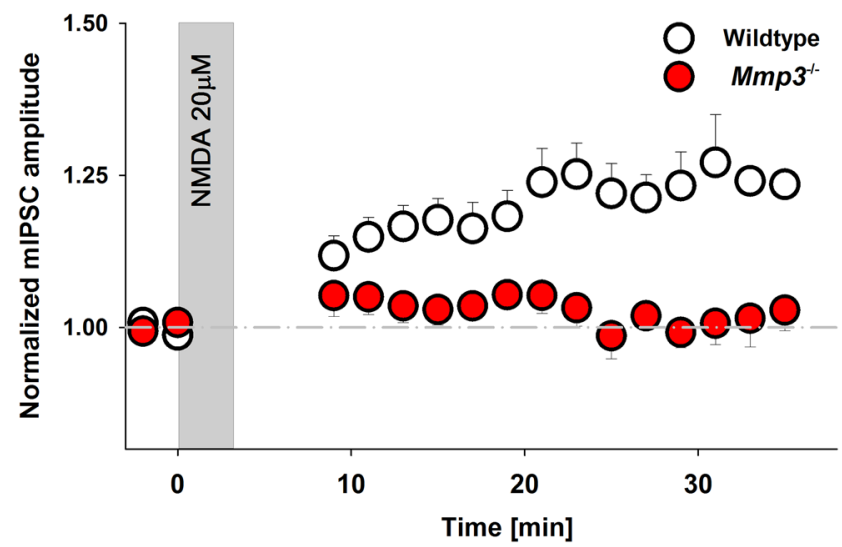

MMP3 activity is crucial for iLTP induction within a narrow time window

Our pharmacological results demonstrated the involvement of MMP3 in the hippocampal iLTP. We next scrutinized the time window during which MMP3 activity is necessary for plasticity induction. To address this issue, we studied the impact of UK-356618 application on iLTP at varying time points after NMDA administration $(3,8$, and 13 min; Fig. 3a). Application of the MMP3 activity inhibitor immediately after the end of plasticity induction led to iLTD $(0.88 \pm 0.02 ; p=0.031$; Wilcoxon signed-rank test in comparison to values before NMDA stimulation; Fig. 3b, c). Interestingly, this effect was similar to the previous one that we observed with UK-356618 application $15 \mathrm{~min}$ before NMDA stimulation (Fig. 1e). It is worth mentioning that we did not observe iLTP when we blocked MMP3 activity 8 min after NMDA stimulation began $(1.06 \pm 0.05$; $p=0.264$, paired $t$ test; Fig. 3b, c). Interestingly, no alterations of iLTP were observed when the MMP3 inhibitor

b

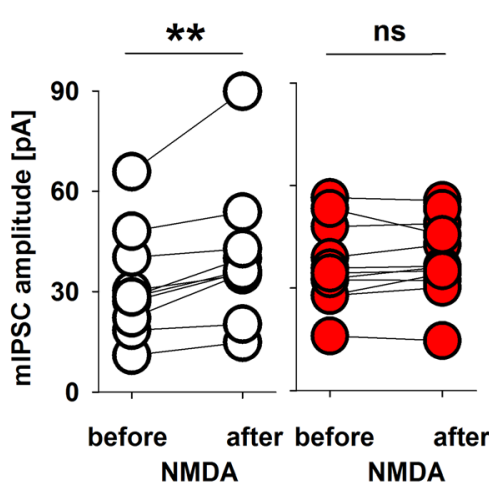

C

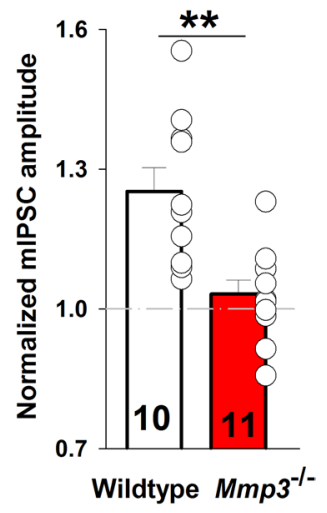

d

Wildtype baseline

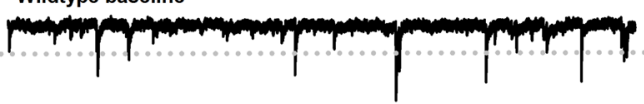

Wildtype after NMDA

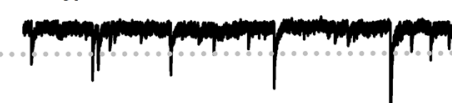

Prom
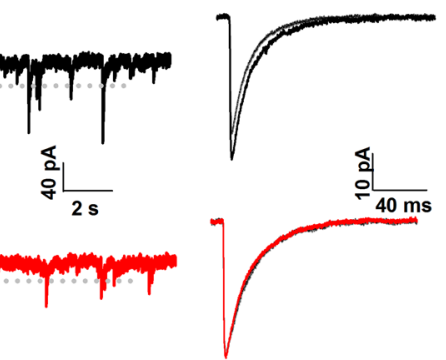

$\mathrm{Mmp3}^{-/-}$baseline

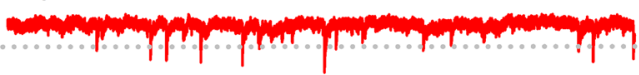

$\mathrm{Mmp3}^{-1-}$ after NMDA

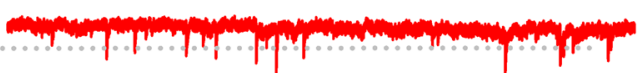

Fig. 2 MMP3 specifically regulates iLTP induction and consolidation. a Time course of iLTP recorded from CA1 pyramidal cells in $\mathrm{Mmp3}^{-1-}$ mice (red) and wild-type mice (white). Note that iLTP was completely abolished in $\mathrm{Mmp}^{-/-}$mice. b Average mIPSC amplitude measured from a single pyramidal cell before and after NMDA stimulation in wild-type slices and $\mathrm{Mmp}^{-/-}$slices (paired $t$ test). c Relative effect of NMDA stimulation on mIPSC amplitude in the wild-type group and $\mathrm{Mmp}^{-/-}$group (unpaired $t$ test). d Examples of raw mIPSC traces recorded before and 20-22 min after iLTP induc- tion in WT (top) and $\mathrm{Mmp}^{-/-}$(bottom) group and the corresponding averaged traces of their amplitude. On the right side grey lines show averaged traces before NMDA stimulation while black and red line shows traces after iLTP induction (in WT and $\mathrm{Mmp}^{-/-}$group respectively). On the left side the dotted line indicates the mean amplitude of baseline mIPSC. In $\mathbf{b}$, the average mIPSC amplitude of each neuron is shown as one circle. $* * p<0.01, * p<0.05$. $n s$ nonsignificant. The numbers on the bars refer to the number of recordings 


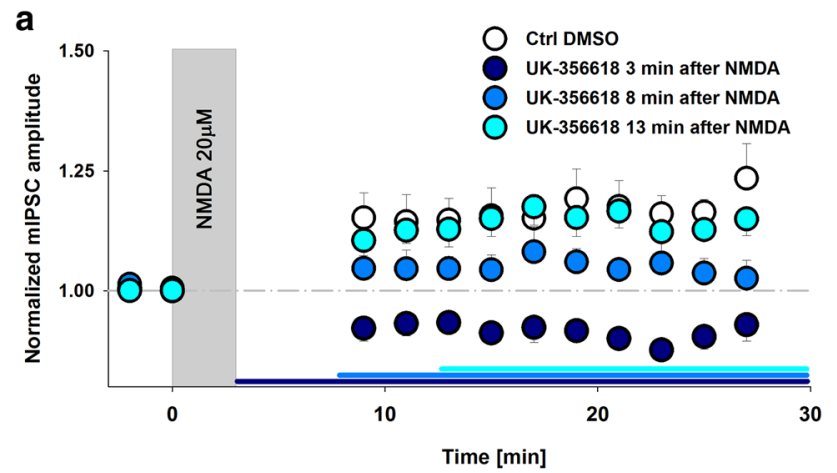

b

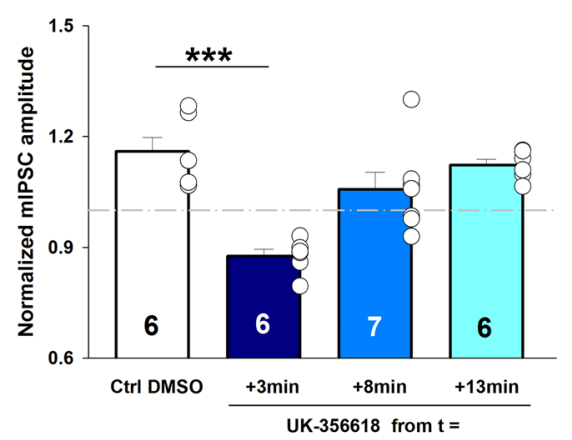

C

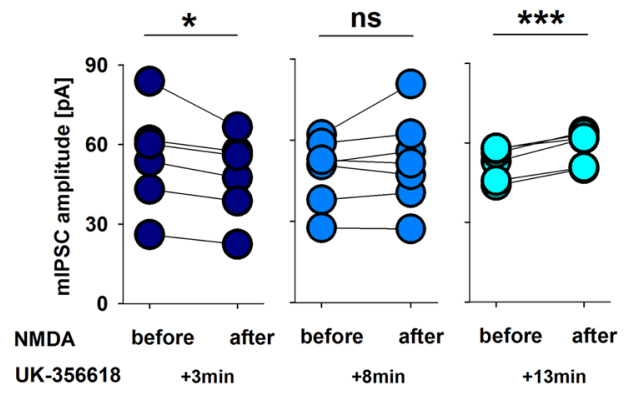

Fig. 3 iLTP requires MMP3 activity within a restricted time window. a Time course of mean mIPSC amplitudes normalized to baseline values in control slices (white) and when UK-355618 was bath-applied at different time points after NMDA stimulation: 3 min (dark blue), 8 min (blue), 13 min (cyan). Note that MMP3 activity was required for iLTP up to $13 \mathrm{~min}$. b Statistics of the ratio of mIPSC amplitudes after/before NMDA stimulation, in control slices (white) and UK-355618-treated slices at the respective time points: 3 min (dark blue), 8 min (blue), 13 min (cyan). The data were analyzed using one-way ANOVA. c Mean mIPSC amplitude recorded from an individual hippocampal neuron before and after NMDA stimulation in the presence of UK-356618 that was applied at varying time points relative to iLTP induction. Notice that UK-356618 that was applied 3 min after iLTP induction led to an iLTP-to-iLTD conversion (dark blue; Wilcoxon signed-rank test). UK-356618 that was applied 8 min after NMDA stimulation abolished iLTP (blue; paired $t$ test). UK-356618 that was applied 13 min after NMDA stimulation had no effect on the size of iLTP (cyan; paired $t$ test). ${ }^{* * *} p<0.001$, ${ }^{*} p<0.05$. $n s$ nonsignificant. The numbers on the bars refer to the number of recorded cells was applied 13 min after NMDA stimulation $(1.12 \pm 0.02$; $p<0.001$, paired $t$ test; Fig. 3b, c). The data presented above show that iLTP requires MMP3 activity up to approximately 13 min after induction.

\section{Inhibition of MMP3 during iLTP induction impairs the recruitment of gephyrin into the synapse}

NMDA-induced iLTP is accompanied by an increase in accumulation of the scaffold protein gephyrin at GABAergic synapses [29]. We thus sought to investigate whether MMP3 inhibition affects synaptic clustering of gephyrin using an immunocytochemical approach. We used cultures of primary hippocampal neurons, which allowed us to analyze distinct discernible clusters of gephyrin in individual dendrites and synapses. To determine whether our major findings could be reproduced in this model, we evaluated iLTP induction and its sensitivity to MMP blockers in cultured neurons. Similar as in slice recording, the MMP2 and MMP9 inhibitor SB-3CT did not affect GABAergic plasticity (Supplementary Fig. 4a-c) while in the presence of UK-356618, cultured neurons did not undergo iLTP (ratio of mIPSC amplitude after/before iLTP; control with DMSO: $1.21 \pm 0.05$; UK-356618: $1.00 \pm 0.03 ; p=0.008$; Fig. $4 \mathrm{a}-\mathrm{c}$ ). We next evaluated NMDA-induced gephyrin modifications by immunolabeling gephyrin and vesicular GABA transporter (vGAT) to reveal GABAergic synapses (Fig. 4d, f). The size and fluorescence intensity of synaptic gephyrin clusters were analyzed under control conditions and $20 \mathrm{~min}$ after iLTP induction, both in wild-type and $\mathrm{Mmp}^{3^{-/}}$mice. As previously reported [30], the bath application of NMDA increased the average size of synaptic gephyrin clusters by $\sim 20 \%$ (area normalized to control; sham: $1.00 \pm 0.02$; iLTP: $1.21 \pm 0.04 ; p<0.001$; Fig. $4 \mathrm{e}$ ), whereas NMDA stimulation in the presence of UK-356618 did not enlarge synaptic gephyrin clusters (sham with UK-356618: 1.00 \pm 0.03 ; iLTP with UK-356618: $0.98 \pm 0.03 ; p=0.64$; Fig. 4e). Furthermore, we observed that in $\mathrm{Mmp}^{-/-}$neurons, the application of NMDA did not cause changes in the size of gephyrin clusters, similar to the UK-356618-treated group $\left(\mathrm{Mmp3}^{-/-}\right.$sham: $1.00 \pm 0.03 ; \mathrm{Mmp3}^{-/-}$iLTP: $1.03 \pm 0.02$; $p=0.50$; Fig. $4 \mathrm{~g}$ ). In parallel, iLTP induction left the average fluorescence intensity of synaptic gephyrin clusters unchanged (Supplementary Fig. 4d, e). These results indicate that functional iLTP is accompanied by structural changes at GABAergic synapses, and both functional and structural manifestations of iLTP can be blocked by inhibiting the proteolytic activity of MMP3. 
a

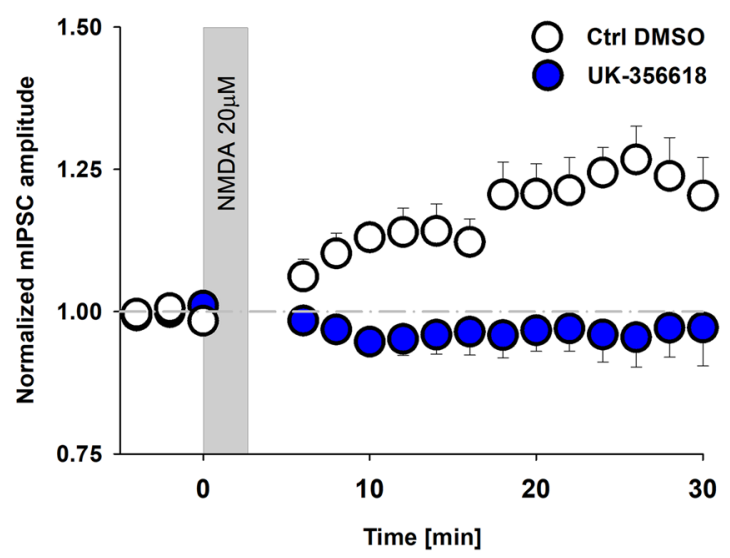

d
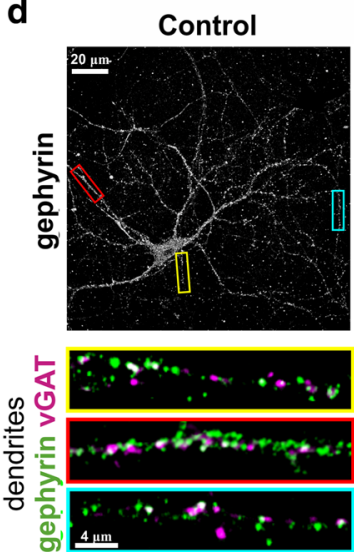

iLTP
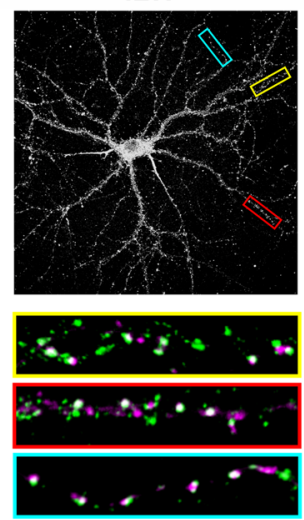

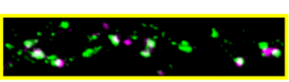

b

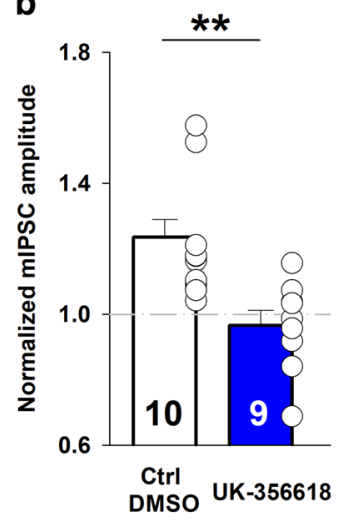

C

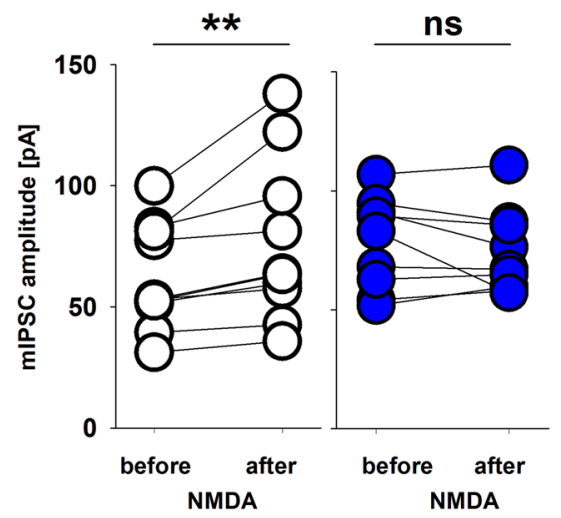

iLTP with UK-356618
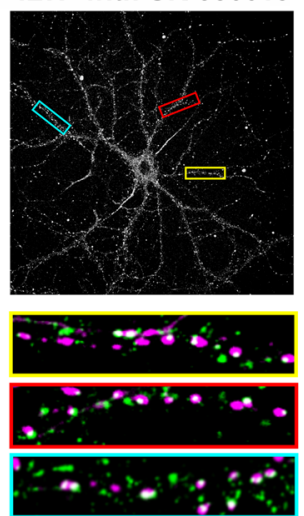

$\mathbf{f}$
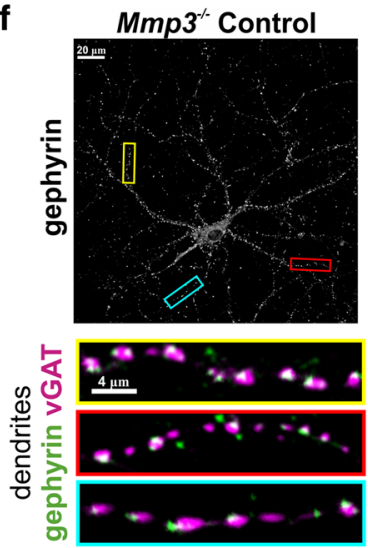

$\mathrm{Mmp3}^{-/}$iLTP
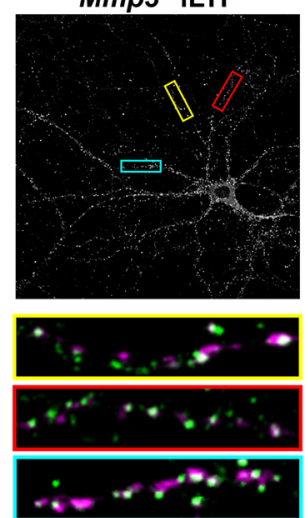

Fig. 4 Inhibition of MMP3 activity blocks iLTP induction and gephyrin accumulation at GABAergic synapses in neuronal cultures. $\mathbf{a}, \mathbf{b}$ Time course (a) and magnitude (b) of NMDA-induced iLTP recorded under control conditions and in the presence of the MMP3 inhibitor UK-356618 ( $t$ test) in cultured hippocampal neurons. The gray area marks the application of NMDA. c Changes in mIPSC amplitude measured before and 20-22 min after iLTP induction with NMDA in the control and UK-356618 groups (paired $t$ test). d, f Representative confocal maximum projection images of neuronal cultures immunolabeled with antibodies against presynaptic vGAT and postsynaptic gephyrin. (Upper) Low-magnification image of the gephyrin signal in the whole neuron. Framed areas are magnified below. (Lower) Magnification of exemplary dendrites indicated above (marked with colored boxes). The colocalization of gephyrin (green) and vGAT (magenta) corresponds to the presence of gephyrin at GABAergic synapses (white). e, $\mathbf{g}$ Quantification of the area of synaptic gephyrin clusters in neurons that were treated with e sham solution, NMDA, UK-356618, and NMDA + UK-356618 (wild type) and g sham solution and NMDA $\left(\mathrm{Mmp3}^{--}\right)$. Values are normalized to respective sham group, $t$ test comparison vs. respective control. ${ }^{*} p<0.01$, $*_{* *} p<0.001$. ns nonsignificant. Numbers on the bars refer to the number of coverslips from at least three different batches of cultures 


\section{Immobilization of GABAA receptors at inhibitory synapses depends on MMP3 activity}

A key mechanism that regulates the number of $\mathrm{GABA}_{\mathrm{A}}$ receptors at inhibitory synapses relies on the ability of synapses to trap receptors that laterally diffuse between synaptic and extrasynaptic domains [31]. At GABAergic synapses, iLTP induction is accompanied by a reduction and more con-

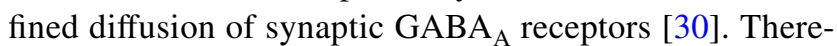
fore, we tested whether the proteolytic activity of MMP3 plays a role in $\mathrm{GABA}_{\mathrm{A}}$ receptor immobilization, which could underlie iLTP in our model. The diffusion of $\mathrm{GABA}_{\mathrm{A}}$ receptors was studied in cultured hippocampal neurons by imaging quantum dots (QDs) that were tethered to the $\alpha 1$ subunit of $\mathrm{GABA}_{\mathrm{A}}$ receptors. Inhibitory synaptic localization was identified by live immunostaining of the presynaptic marker vGAT.

We compared the diffusion properties of synaptic $\mathrm{GABA}_{\mathrm{A}}$ receptors before and $20 \mathrm{~min}$ after iLTP induction with NMDA. This analysis revealed that sham treatment (i.e., only vehicle treatment) did not affect the diffusion of $\alpha_{1} \mathrm{GABA}_{\mathrm{A}}$ receptors, the fraction of immobile receptors, or the mean squared displacement (MSD; Supplementary Fig. 5a-c). In contrast, comparisons of MSD plots before and 20 min after NMDA stimulation indicated that iLTP induction resulted in a more confined diffusion of synaptic $\alpha_{1} \mathrm{GABA}_{\mathrm{A}}$ receptors (Fig. 5a, b) [29]. iLTP induction with NMDA significantly slowed synaptic $\mathrm{GABA}_{\mathrm{A}}$ receptors, reflected by a lower diffusion coefficient (median and interquartile range [IQR] before: $0.0124,0.0018-0.0393$ $\mu \mathrm{m}^{2} \mathrm{~s}^{-1}$; median and IQR after: 0.0047, 0.0006-0.0190 $\mu \mathrm{m}^{2} \mathrm{~s}^{-1} ; p<0.001$; Fig. $5 \mathrm{c}$ ) and a prolonged duration of immobile periods (immobile fraction) (before: $0.41 \pm 0.03$; after: $0.61 \pm 0.03 ; p<0.001$; Fig. 5 d). To further test the effect of MMP3 inhibition on $\mathrm{GABA}_{\mathrm{A}}$ receptor diffusion, we induced iLTP in the presence of UK-356618. The inhibition of MMP3 activity prevented the increase of confinement of synaptic trajectories (Fig. 5e, f), prevented the reduction of the diffusion coefficient (median and IQR before: 0.0094, 0.0013-0.0270 $\mu^{2} \mathrm{~s}^{-1}$; median and IQR after: $0.0138,0.0022-0.0384 \mu \mathrm{m}^{2} \mathrm{~s}^{-1} ; p=0.24$; Fig. $5 \mathrm{~g}$ ) and prevented the increase of immobilization of $\mathrm{GABA}_{\mathrm{A}}$ receptors (immobile fraction before: $0.48 \pm 0.03$; after: $0.42 \pm 0.02$; $p=0.41$; Fig. $5 \mathrm{~h}$ ), and, thus leaving receptor lateral diffusion unaffected by the iLTP induction protocol. Treatment with UK-356618 alone (without NMDA) did not affect the diffusion properties of synaptic $\alpha_{1} \mathrm{GABA}_{\mathrm{A}}$ receptors (Supplementary Fig. $5 d-f$ ). These results suggest that the lateral diffusion of $\mathrm{GABA}_{\mathrm{A}}$ receptors during the iLTP is modulated by MMP3-mediated extracellular proteolytic activity.

\section{Exogenous active MMP3 induces plasticity at GABAergic synapses}

Considering that inhibition of endogenous MMP3 blocked the expression of postsynaptic GABAergic iLTP, we next investigated whether exogenous active recombinant rMMP3 affects inhibitory synaptic transmission. Brief treatment for 3 min (see "Methods" sections for a description of used treatment durations) with active rMMP3 (400 ng/ml) in neuronal cultures progressively increased the mIPSC amplitude, which reached on average $119 \%$ of baseline after $28-30 \mathrm{~min}$ (Fig. 6a-c). Comparisons of mIPSC amplitudes before and 28-30 min after incubation with rMMP3 indicated significant potentiation (before: $47.6 \pm 5 \mathrm{pA}$; after: $56.0 \pm 5 \mathrm{pA}$; $p=0.002$; Fig. $6 \mathrm{~d}$ ) that was maintained throughout the rest of the recording period (up to $60 \mathrm{~min}$ in the most stable recordings; Supplemental Fig. 6a). In the sham group (i.e., treated only with vehicle), we did not observe changes in mIPSC amplitude (before: $64.7 \pm 7 \mathrm{pA}$; after: $63.9 \pm 8 \mathrm{pA} ; p=0.74$; Fig. 6d). Neither sham treatment nor rMMP3 application changed the mIPSC frequency (Supplemental Fig. 6b). Altogether, these results indicate that MMP3 activity induces the potentiation of inhibitory synaptic transmission that lasts for at least $1 \mathrm{~h}$. Such potentiation may be regarded as chemically induced LTP and referred to as MMP3-iLTP.

We next investigated whether the diffusion of synaptic $\mathrm{GABA}_{\mathrm{A}}$ receptors is directly modulated by exogenous MMP3 activity. We performed single-particle tracking of $\alpha_{1} \mathrm{GABA}_{\mathrm{A}}$ receptors in neurons that were acutely treated for 2 min with active rMMP3 (Fig. 6e; see "Methods" section for a description of used treatment durations). The MSD vs. time plot of synaptic trajectories reached a lower plateau after MMP3 treatment, suggesting more confined $\alpha_{1} \mathrm{GABA}_{\mathrm{A}}$ receptor diffusion (Fig. 6f). Furthermore, the median of the diffusion coefficient of synaptic receptors changed from $0.0069 \mu^{2} \mathrm{~s}^{-1}$ before to 0.0022 $\mu \mathrm{m}^{2} \mathrm{~s}^{-1} 20 \mathrm{~min}$ after MMP3 treatment (IQR before: $0.00075-0.0252 \mu \mathrm{m}^{2} \mathrm{~s}^{-1}$, after: 0.00052-0.0194 $\mu^{2} \mathrm{~s}^{-1}$; $p=0.008$; Fig. 6g). After rMMP3 application, the distribution of diffusion coefficients shifted to significantly lower values, with a higher immobile fraction (before: $0.53 \pm 0.03$, after: $0.61 \pm 0.03 p=0.018$; Supplemental Fig. 7b) and a longer residence time at the synapse (before: $22 \pm 1 \mathrm{~s}$; after: $27 \pm 1 \mathrm{~s} ; p=0.011$; Fig. $6 \mathrm{~h}$ ). This result was corroborated by matched observations of the same $\mathrm{GABA}_{\mathrm{A}}$ receptors identified at the synapse before and $20 \mathrm{~min}$ after rMMP3 infusion, indicating a significant decrease in the diffusion coefficient (Supplemental Fig. 7a). The diffusion parameters of extrasynaptic $\alpha_{1} \mathrm{GABA}_{\mathrm{A}}$ receptors were comparable before and after MMP3 treatment (Supplemental Fig. 7c, d). These results indicate that the proteolytic activity of MMP3 promotes the synaptic trapping of 

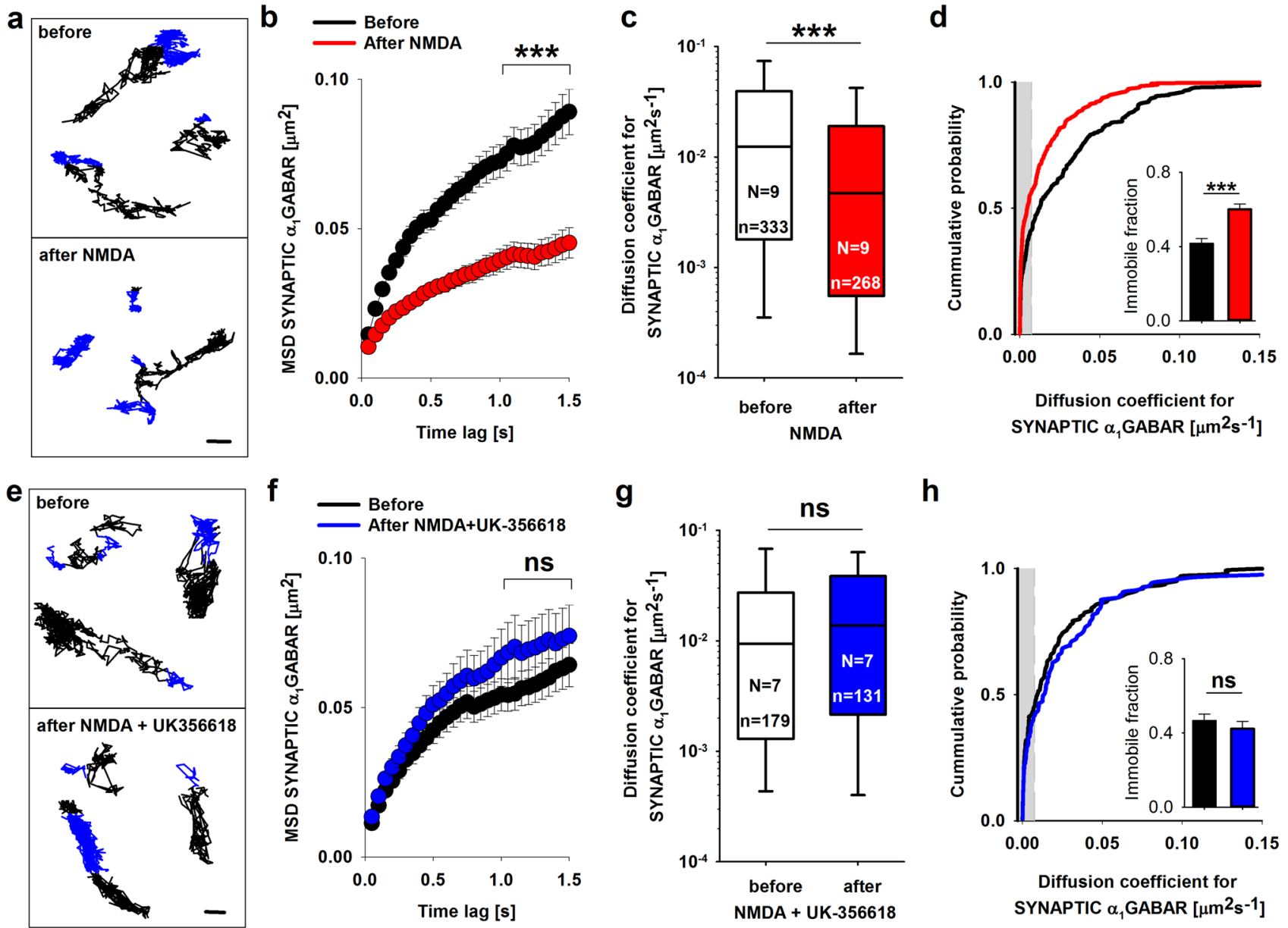

h

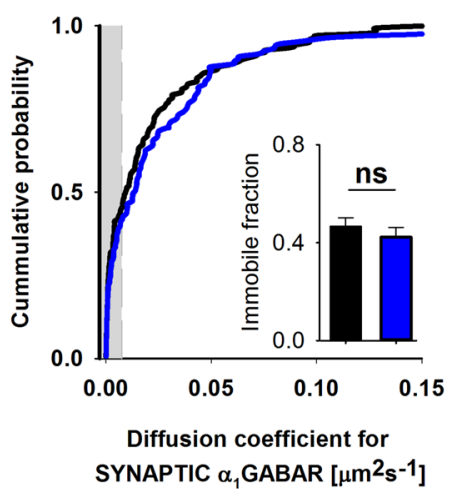

Fig. 5 Immobilization of $\alpha_{1} \mathrm{GABA}_{\mathrm{A}}$ receptors at GABAergic synapses critically depends on MMP3 activity. a Representative trajectories of individual $\alpha_{1}$-containing $\mathrm{GABA}_{\mathrm{A}}$ receptors diffusing in extrasynaptic membrane (black) and in synaptic region (blue) identified by live vGAT staining. Scale bar $500 \mathrm{~nm}$. b Mean square displacement of synaptic $\alpha_{1} \mathrm{GABA}_{\mathrm{A}}$ receptors before (black) and $20 \mathrm{~min}$ after (red) NMDA stimulation. $\mathbf{c}$ Interquartile range (IQR; 25-75\%) and median diffusion coefficient of synaptic $\alpha_{1} \mathrm{GABA}_{\mathrm{A}}$ receptors before and 20 min after NMDA stimulation. d Cumulative probability distributions of diffusion coefficients for synaptic $\alpha_{1} \mathrm{GABA}_{\mathrm{A}}$ receptors before (black) and $20 \mathrm{~min}$ after (red) NMDA stimulation. The gray area marks the part of the cumulative distribution that contains immobile receptors $\left(D<0.0075 \mu \mathrm{m}^{2} \mathrm{~s}^{-1}\right)$. (Inset) Comparison of the immobile fraction before and after iLTP. e Representative trajectories of individual $\alpha_{1}$-containing $\mathrm{GABA}_{\mathrm{A}}$ receptors diffusing in extrasynaptic membrane (black) and in synaptic region (blue) acquired before and

$\mathrm{GABA}_{\mathrm{A}}$ receptors, resulting in potentiation of the GABAergic synapse.

\section{Potentiation induced by active rMMP3 occludes NMDA-induced iLTP}

To further investigate the effect of exogenous rMMP3 activity on inhibitory synapses, we analyzed the size and average fluorescence intensity of synaptic gephyrin clusters (Fig. 6i).

20 min after MMP3 infusion. Scale bar $500 \mathrm{~nm}$. f Mean square displacement of synaptic $\alpha_{1} \mathrm{GABA}_{\mathrm{A}}$ receptors before (black) and $20 \mathrm{~min}$ after (blue) NMDA stimulation with UK-356618. g Interquartile range and median diffusion coefficient of synaptic $\alpha_{1} \mathrm{GABA}_{\mathrm{A}}$ receptors before and $20 \mathrm{~min}$ after NMDA stimulation in the presence of UK356618. h Cumulative probability distributions of diffusion coefficients for synaptic $\alpha_{1} \mathrm{GABA}_{\mathrm{A}}$ receptors before (black) and $20 \mathrm{~min}$ after (blue) NMDA stimulation in the presence of UK-356618. The gray area marks the part of the cumulative distribution that contains immobile receptors $\left(D<0.0075 \mu^{2} \mathrm{~s}^{-1}\right)$. (Inset) Comparison of the immobile fraction before and after iLTP induced in the presence of UK-356618. $* * * p<0.001$. $n s$ nonsignificant. The data in $\mathbf{b}, \mathbf{c}, \mathbf{d}-$ inset, $\mathbf{f}, \mathbf{g}$, and $\mathbf{h}$-inset, were analyzed using the Mann-Whitney $U$ test. $N$ and $n$ refer to the number of coverslips and analyzed trajectories, respectively

Active rMMP3 application for 2 min $15 \mathrm{~s}$ increased the mean size of synaptic (i.e., vGAT-positive) gephyrin clusters compared with sham-treated neurons (area normalized to control; sham: $1.00 \pm 0.02$; MMP3-iLTP: $1.08 \pm 0.03 ; p=0.029$; Fig. 6j). rMMP3 treatment decreased also the average fluorescence intensity of gephyrin synaptic clusters (Supplemental Fig. 7e). Overall, the effect of exogenous active rMMP3 on the area of gephyrin clusters further substantiates 


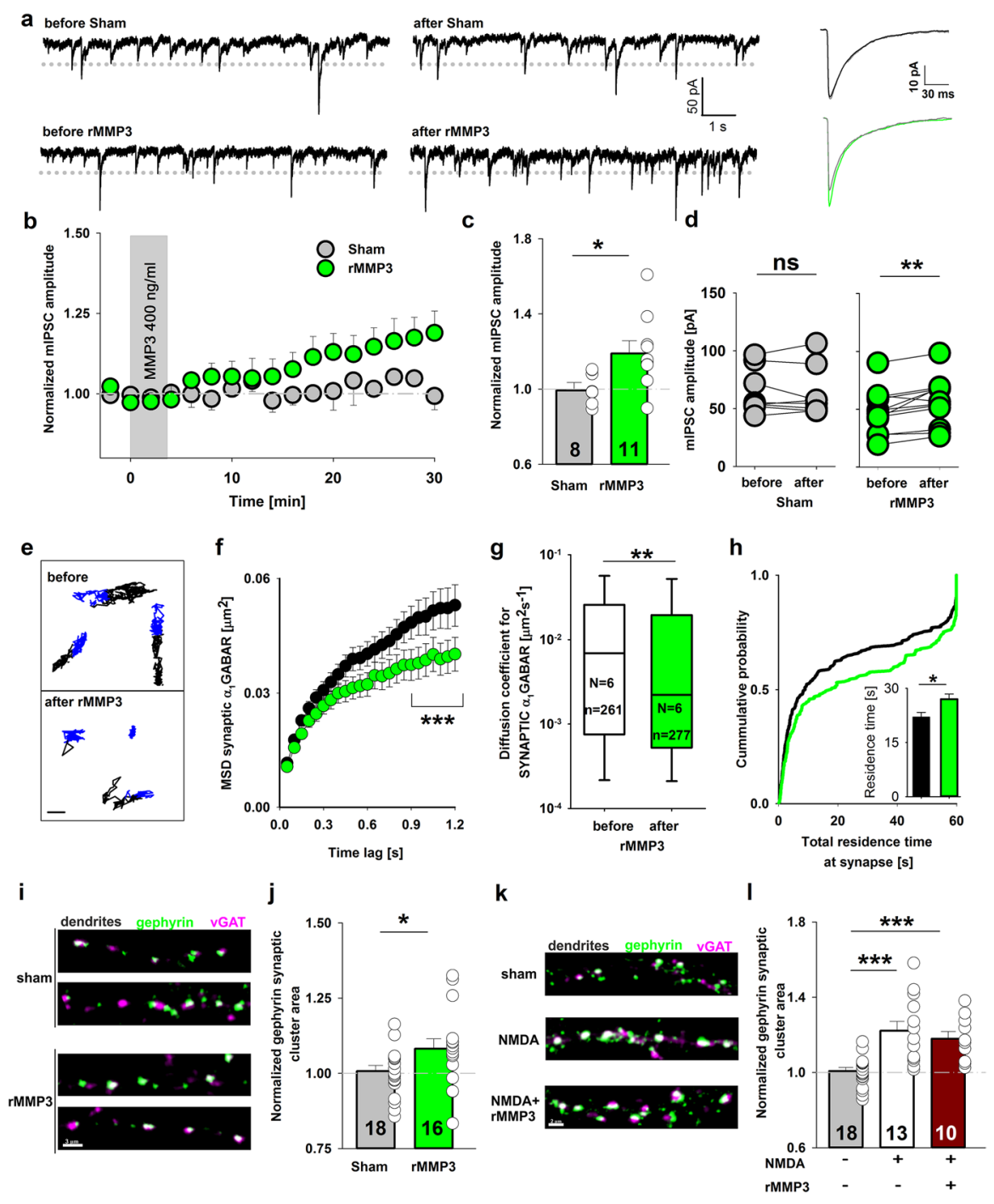

Fig. 6 Short-term application of active rMMP3 potentiates mIPSC amplitude, immobilizes synaptic GABA receptors and increases the size of synaptic gephyrin clusters. a On left: typical raw mIPSC recordings before and $30 \mathrm{~min}$ after $\mathrm{rMMP} 3$ or sham treatment. Dotted line represents the man amplitude of baseline mIPSC. On right: averaged mIPSC traces recorded before (grey) and $30 \mathrm{~min}$ after treatment (sham-black, rMMP3 - green). b mIPSC amplitude recorded in the sham-treated group and in neurons that were incubated for 3 min with active rMMP3 (400 ng/ml). c Comparison of changes in mIPSC amplitude in the sham group and in neurons that were treated with rMMP3. d Changes in mIPSC amplitude measured before and 28-30 min after rMMP3 administration (green) or sham treatment (gray). e $\alpha_{1} \mathrm{GABA}_{\mathrm{A}}$ receptor trajectories before and after application of rMMP3. Extrasynaptic parts of trajectories are marked as black, synaptic parts are marked blue. Scale bar $500 \mathrm{~nm}$. f Mean square displacement of synaptic $\alpha_{1} \mathrm{GABA}_{\mathrm{A}}$ receptors before (black) and 20 min after (green) rMMP3 incubation. g Interquartile range and median diffusion coefficient of synaptic $\alpha_{1} \mathrm{GABA}_{\mathrm{A}}$ receptors before and $20 \mathrm{~min}$ after short-term ( $2 \mathrm{~min}$ ) incubation with rMMP3. $N$ and $n$ refer to the number of coverslips and analysed trajectories, respectively. $\mathbf{h}$ Cumulative probability distributions of the total residence time in the synapse by $\alpha_{1} \mathrm{GABA}_{\mathrm{A}}$ receptors before (black) and 20 min after (green) the application of rMMP3. The total imaging time $(1 \mathrm{~min})$ indicates the maximal possible time that the analysed

receptors could be detected in the synapse. (Inset) Average total residence time before and after rMMP3 treatment. i Representative confocal maximum projection images obtained from neuronal cultures that were immunolabeled with antibodies against presynaptic vGAT (magenta) and postsynaptic gephyrin (green). The colocalization of gephyrin and vGAT (white) corresponds to the presence of gephyrin at GABAergic synapses. (Upper) Images of two typical portions of dendrites after sham treatment. (Lower) Images of two typical portions of dendrites that undergo 2 min $15 \mathrm{~s}$ incubation with rMMP3 (400 ng/ml). $\mathbf{j}$ The average area of gephyrin clusters at dendritic inhibitory synapses (normalized to sham). k Representative confocal maximum projection images obtained from neuronal cultures that were labeled with antibodies against presynaptic vGAT (magenta) and postsynaptic gephyrin (green). The upper, middle, and lower images show typical fragments of dendrites that were fixed $20 \mathrm{~min}$ after sham, NMDA, and NMDA+rMMP3 treatment, respectively. 1 Quantification of the average area of synaptic gephyrin clusters in neuronal cultures that were treated with sham solution, NMDA, and $\mathrm{NMDA}+\mathrm{rMMP} 3$. Values are normalized to sham group. ${ }^{*} p<0.05$, $* * p<0.01, * * * p<0.001$. ns nonsignificant. The data in $\mathbf{c}, \mathbf{j}, \mathbf{l}$, were analyzed using $t$ test vs. respective control. The data in $\mathbf{d}$ were analyzed using paired $t$ tests. The data in $\mathbf{f}-\mathbf{h}$ were analyzed using the Mann-Whitney $U$ test. Numbers on the bars refer to the number of coverslips from at least three different batches of cultures 
the notion that extracellular proteolysis might regulate the strength of GABAergic synapses.

In the next series of experiments, we investigated the relationship between NMDA-induced iLTP and MMP3-iLTP to determine whether MMP3-iLTP occludes NMDA-induced iLTP. We reasoned that if MMP3-iLTP and NMDA-induced iLTP arise from two separate molecular mechanisms, then we should observe an additive effect on gephyrin cluster area when both NMDA and MMP3 are applied together. However, the application of NMDA together with exogenous rMMP3 increased the size of synaptic gephyrin clusters to a similar level as it was observed after treatment with NMDA alone (area normalized to control; sham: $1.00 \pm 0.02$; NMDA: $1.22 \pm 0.05$; NMDA +rMMP3: $1.18 \pm 0.04$; NMDA $v s$. NMDA + rMMP3 $p=0.528$; Fig. 6k, i). Neither NMDA alone nor NMDA + rMMP3 altered the average fluorescence intensity of gephyrin synaptic clusters (Supplemental Fig. 7f). These results suggest that both the activity of NMDA receptors and MMP3-dependent proteolysis participate in the same molecular mechanism that is responsible for iLTP induction.

\section{Exogenous rMMP3 rescues impairment in iLTP in MMP3-deficient neurons}

To study the impact of MMP3 excess on GABAergic plasticity we applied exogenous active rMMP-3 and examined NMDA-iLTP in wild-type and $\mathrm{Mmp}^{-/-}$neuronal cultures. Synaptic recordings from cultured hippocampal neurons prepared from $\mathrm{Mmp}^{-/-}$mice indicated that MMP3 deficiency abolished NMDA-induced iLTP (ratio of mIPSC amplitude after/before iLTP; wild type: $1.22 \pm 0.05 ; \mathrm{Mmp}^{-/-}$: $1.01 \pm 0.03 ; p=0.001 ;$ Fig. $7 \mathrm{a})$, similar to hippocampal slices (Fig. 2a). We next examined whether exogenous active rMMP3 application together with NMDA (for $2 \mathrm{~min}$ $15 \mathrm{~s})$ restores iLTP in $\mathrm{Mmp3}^{-/-}$mice. The administration of active rMMP3 during NMDA application restored iLTP in $\mathrm{Mmp3}^{-/-}$neurons $\left(\mathrm{Mmp3}^{-/-}\right.$+ rMMP3 + NMDA: $1.29 \pm 0.07$; vs. $M m p 3^{-/-}$from Fig. 7a; $p<0.001$; Fig. 7b, c). Next, we evaluated the effect of rMMP3 application together with NMDA in wild-type cultures. Stimulation with NMDA induced stable iLTP in wild-type neurons (Fig. 7a), but the simultaneous application of NMDA and rMMP3 in this group for 2 min $15 \mathrm{~s}$ did not further increase iLTP; instead, it significantly reduced it (wild-type + rMMP3 + NMDA: $1.06 \pm 0.05 ;$ vs. wild-type NMDA-iLTP form Fig. 7a; $p=0.029$; Fig. $7 \mathrm{~b}, \mathrm{c}$ ). Thus, the ability of $\mathrm{Mmp3}^{-/-}$neurons to induce iLTP was restored by exogenous rMMP3, whereas when the exogenous protease was applied together with NMDA in wild-type neurons, an impaired NMDA-iLTP was observed suggesting that excess of MMP3 activity is detrimental for GABAergic plasticity (Fig. 7d). Based on these data, it may be proposed that effective iLTP requires fine-tuned MMP3 activity.

The duration of NMDA application determines the direction of GABAergic plasticity. Moderate postsynaptic $\mathrm{Ca}^{2+}$ influx drives iLTP [32], and much stronger NMDA receptor activation results in iLTD [33]. We hypothesized that NMDAR activation that is adequate for iLTP induction in the wild-type group may be insufficient in $\mathrm{Mmp}^{-/-}$neurons. We thus extended the duration of NMDA application from $2 \min 15 \mathrm{~s}$ to $2 \min 45 \mathrm{~s}$, but this longer duration did not result in iLTP induction in wild-type or $\mathrm{Mmp3}^{-/-}$neurons (Supplemental Fig. 8a-c). Similarly, the prolonged NMDA stimulation did not rescue the curtailed structural plasticity of the synaptic gephyrin area in the presence of UK-356618 (Supplemental Fig. 8d-f). Thus, iLTP impairment that was caused by MMP3 deficiency cannot be explained by diminished NMDA receptor activation because it was not restored by prolonged NMDA stimulation.

\section{MMP3 deficiency enhances hippocampus-dependent learning and memory}

To investigate the possible behavioral role of MMP3 we sought to assess hippocampus-dependent spatial learning and memory in $\mathrm{Mmp3}^{-/-}$mice using the Morris water maze. Both $\mathrm{Mmp3}^{-/-}$and wild-type animals were trained in four trials per day for 10 days in the hidden-platform version of the Morris water maze. As training progressed, the path length (i.e., a measure of the total distance travelled to reach the platform) decreased in both wild-type and $M m p 3^{-1-}$ mice (Fig. 8a). Path length was chosen as a performance measure because it reflects cognitive function in the Morris water maze [34]. The two-way analysis of variance (ANOVA) indicated a significant main effect of genotype on learning curves $\left(F_{1,180}=5.35, p=0.022\right)$. Interestingly, in $M m p 3^{-/-}$mice, the path length decreased more rapidly over consecutive trials, suggesting faster learning, reflected by the average day 2 swimming distance to reach the platform (wild type: $892 \pm 88 \mathrm{~cm} ; M m p 3^{-l-}: 541 \pm 54 \mathrm{~cm} ; p=0.0047$; Fig. 8b). To test spatial reference memory, a probe trial without the platform was performed on days 8 and 15, but no difference in the time spent in the target quadrant was found between genotypes (probe 1: genotype $\times$ distance interaction, $F_{3,72}=1.29, p=0.29$, Fig. $8 \mathrm{c}$; probe 2 : genotype $\times$ distance interaction, $F_{372}=0.77, p=0.52$; Fig. $8 \mathrm{~d}$ ). Thus, spatial learning in the Morris water maze progressed faster in $\mathrm{Mmp3}^{-/-}$mice. After completion of the learning trials, however, $\mathrm{Mmp3}^{-1-}$ mice had the same spatial reference memory score as wild-type controls.

In the next series of behavioral experiments, we evaluated the contribution of MMP3 to the associative learning of conditioned fear. Within this experiment baseline freezing in the test cage was recorded on day 1 (Fig. 8e). On 

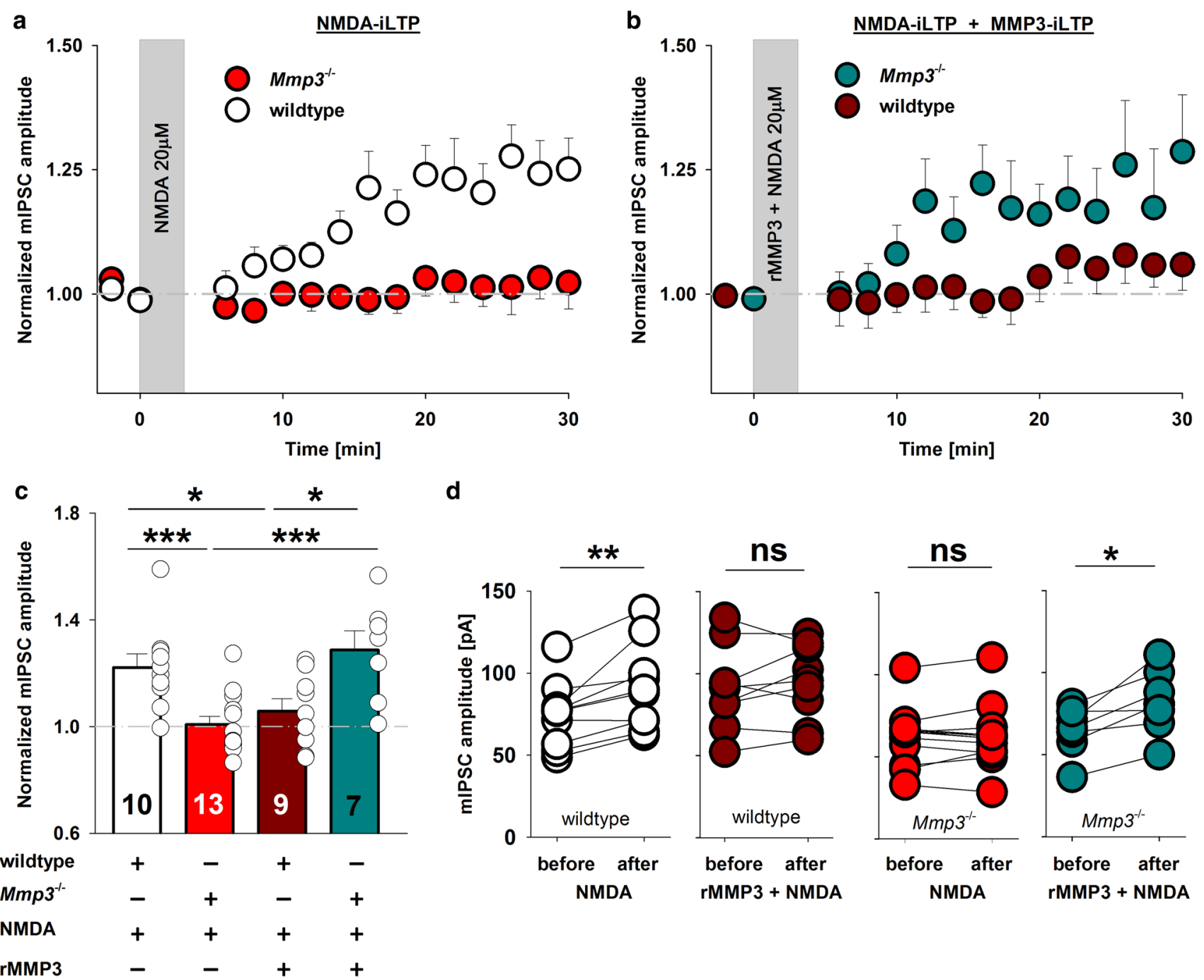

d
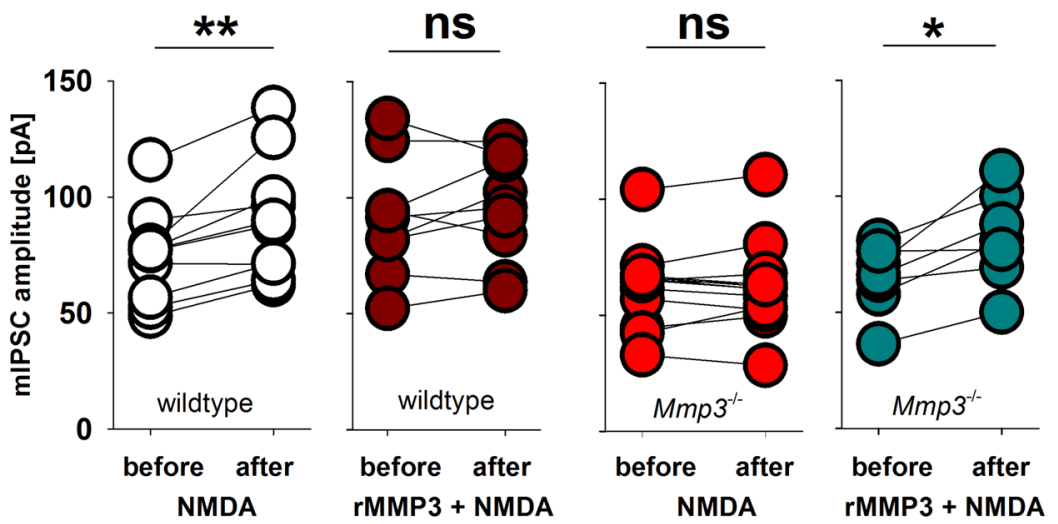

Fig. 7 Short-term application of exogenous active rMMP3 together with NMDA restores iLTP in $M m p 3^{---}$neurons and impairs iLTP in wild-type neuronal cultures. a Time course of NMDA-induced iLTP recorded in hippocampal neuronal cultures that were prepared from wild-type (white) and $\mathrm{Mmp3}^{-/-}$(red) mice. The gray area marks the application of NMDA. b Time course of iLTP that was induced with NMDA coapplied with active rMMP3 $(400 \mathrm{ng} / \mathrm{ml})$ for $2 \mathrm{~min} 15 \mathrm{~s}$ in hippocampal neuronal cultures from wild-type (brown) and $M m p 3^{-/-}$

day 2, footshocks were paired with the spatial context of the test cage and an acoustic signal. Upon returning the animals to the test cage on day 3 , memory of the spatial context was examined by analyzing the freezing response. Finally, the context of the test cage was modified to measure freezing in a different spatial context. The mice were tested for cued fear memory by analyzing freezing in response to the acoustic signal. Both wild-type and $\mathrm{Mmp}^{3^{-/}}$mice exhibited similar freezing during habituation and during the acquisition phase of fear conditioning, suggesting the lack of an anxiety-related phenotype and no short-term or working memory deficits. Remarkably, the $\mathrm{Mmp3}^{-/-}$mice (dark cyan) mice. The gray area marks the application of NMDA with MMP3. c Comparison of changes in mIPSC amplitude after iLTP induction in the respective groups (colors as in $\mathbf{a}$ and b). $\mathbf{d}$ mIPSC amplitude measured before and 20-22 $\mathrm{min}$ after iLTP induction (colors as in $\mathbf{a}$ and $\mathbf{b}$ ). ${ }^{*} p<0.05, * * p<0.01, * * * p<0.001$. The data in $\mathbf{c}$ were analyzed using one-way ANOVA. The data in $\mathbf{d}$ were analyzed using paired $t$ test. The numbers on the bars refer to the number of coverslips from at least three different batches of cultures

exhibited a significant increase in freezing during the context-related memory retention test $24 \mathrm{~h}$ after learning (wild type: $47 \pm 6.7 \%$ freezing time; $M m p 3^{-/-}: 73 \pm 4.6 \%$ freezing time; $p=0.0053$; Fig. 8e). Additionally, $\mathrm{Mmp3}^{-/-}$and wild-type mice exhibited similar freezing in the modified spatial context on day 3 (wild type: $27 \pm 5.1 \%$ freezing time; $M m p 3^{-/-}: 31 \pm 6.4 \%$ freezing time; $p=0.70$ ) and exhibited a normal freezing response to the acoustic signal, suggesting no changes in amygdala-dependent cued fear conditioning (wild type: $65 \pm 8.6 \%$ freezing time; $\mathrm{Mmp}^{-1-}: 75 \pm 5.0 \%$ freezing time; $p=0.34$; Fig. $8 \mathrm{e}$ ). These behavioral findings suggest that the lack of MMP3 activity accelerates spatial 


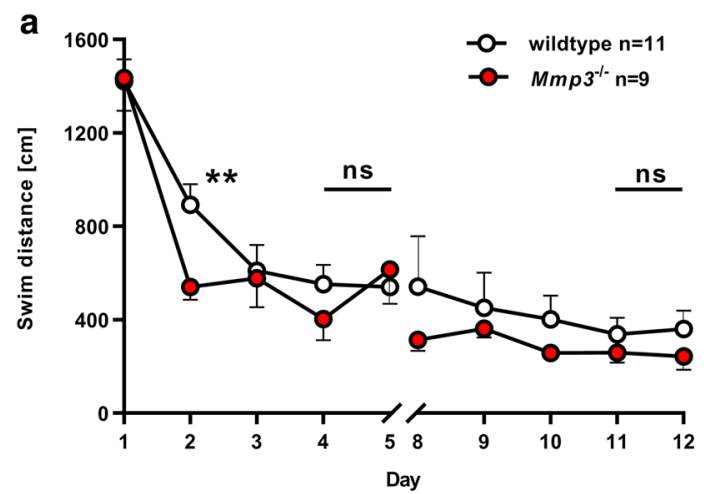

d

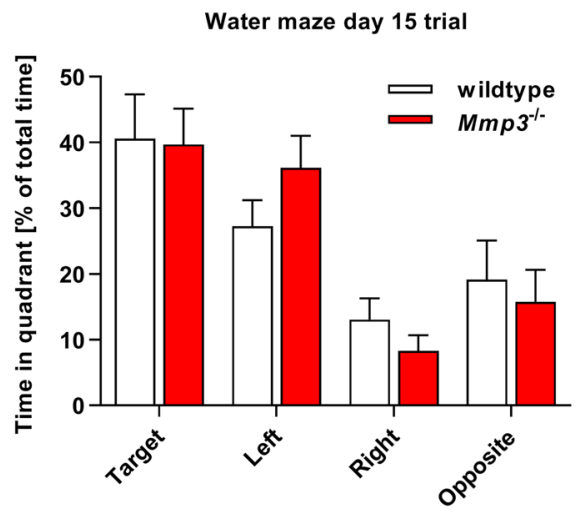

Fig. $8 \mathrm{Mmp3}^{-/-}$mice exhibit faster spatial learning and enhanced contextual fear memory. a-d $\mathrm{Mmp}^{-/-}$mice exhibited a significant difference in spatial learning in the Morris water maze test without changes in memory retrieval. a Swim distance to reach the hidden platform during a 10-day learning period. b Comparison of swim distances to reach the platform on day 2 between wild-type and $\mathrm{Mmp}^{3^{--}}$mice. c, $\mathbf{d}$ Percent time spent in each of the quadrants during the probe memory trials on day 8 (c) and day 15 (d). e Experimental design for measurements of contextual and cued fear memory. Different arrow colors indicate distinct experimental cages (spatial

learning in the Morris water maze and enhances spatial associative fear memory.

\section{Discussion}

In the present study, we found that MMP3-dependent extracellular proteolysis plays a central role in postsynaptic GABAergic plasticity that is induced heterosynaptically by short-lasting NMDA stimulation. Our finding of the crucial role of MMP3 activity in this form of inhibitory plasticity was confirmed both at functional and structural level using various experimental approaches. First, pharmacological inhibition or genetic deficiency of MMP3
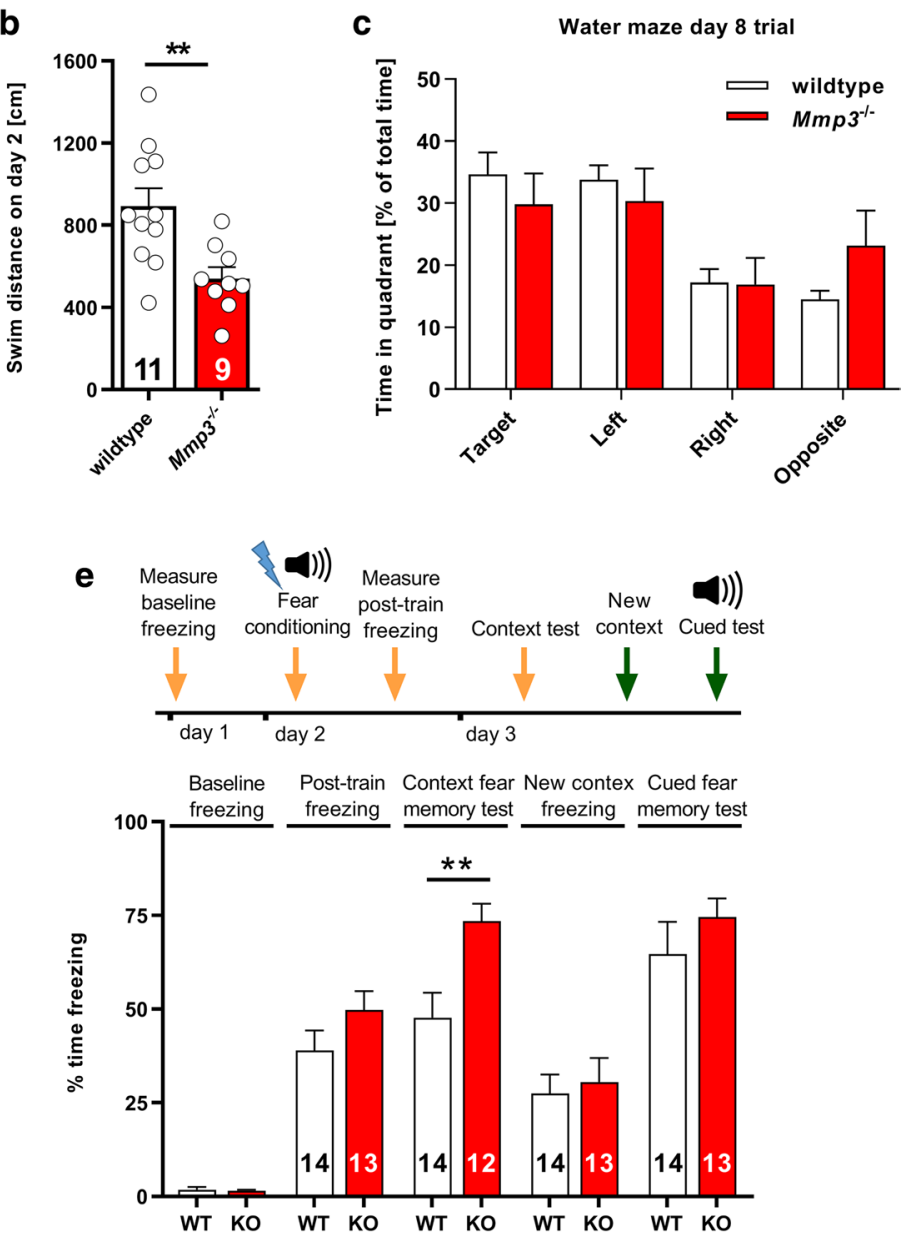

contexts). $\mathrm{Mmp3}^{-/-}$mice (KO) exhibited a significant enhancement of contextual fear memory $24 \mathrm{~h}$ after contextual fear conditioning in comparison to wild-type mice (WT). $\mathrm{Mmp3}^{-/-}$mice exhibited no significant changes in basal freezing, freezing during fear conditioning, or freezing in the new context (pre-CS). $\mathrm{Mmp3}^{-l-}$ mice exhibited similar cue-related memory retention. ${ }^{* *} p<0.01$. The data in a, $\mathbf{c}, \mathbf{d}$, and $\mathbf{e}$ were analyzed using two-way ANOVA. The data in $\mathbf{b}$ and $\mathbf{c}$ for the single comparison between genotypes were analyzed using $t$ tests. The numbers on the bars indicate the number of animals

completely abolished iLTP. Second, the expression of iLTP was accompanied by a larger area of synaptic gephyrin clusters, whereas this phenomenon was not observed when this induction protocol was applied in the presence of the MMP3 inhibitor or in $\mathrm{Mmp}^{-/-}$neurons. Importantly, the structural reorganization of inhibitory synapses upon iLTP induction has been consistently shown to be a crucial determinant of plastic changes in the present model [29, 35]. MMP3 inactivation affected both functional and structural aspects of postsynaptic iLTP, underscoring the key role of this enzyme and suggesting that it might be involved in molecular mechanisms of iLTP at their early stages. Third, 20 minutes after iLTP induction, $\alpha_{1} \mathrm{GABA}_{\mathrm{A}}$ receptors were characterized by slower and more constrained diffusion within inhibitory 
synapses. Conversely, when MMP3 was blocked, the lateral movement of $\mathrm{GABA}_{\mathrm{A}}$ receptors was comparable to that before NMDA application (Fig. 5). Thus, the key mechanism of trapping $\mathrm{GABA}_{\mathrm{A}}$ receptors within the synaptic densities, whereby iLTP is being built up in this model, critically depends on MMP3 activity. Fourth, the central role of MMP3 in GABAergic plasticity was further demonstrated by the fact that the major manifestations of iLTP (i.e., increase in mIPSC amplitude, enlargement of synaptic gephyrin clusters, and the slower lateral diffusion of synaptic $\alpha_{1} \mathrm{GABA}_{\mathrm{A}}$ receptors) were evoked by exogenous, short (2-3 min) administration of active rMMP3. This observation further suggests that MMP3 is involved in mechanisms of iLTP induction at early stages, which was corroborated by the finding that MMP3 is operative during iLTP induction only within a relatively narrow time window (approximately $10 \mathrm{~min}$ ). Furthermore, increased area and decreased average fluoresce intensity of gephyrin clusters after rMMP3 application suggest gephyrin dispersal or redistribution within the synapse. Finally, no additive effects of NMDA and MMP3 on the size of gephyrin synaptic clusters were found (Fig. 6). The application of NMDA and rMMP3 together in wildtype neurons weakened functional iLTP (mIPSC amplitude) despite the increase in gephyrin cluster area to the level observed after NMDA-iLTP or MMP3-iLTP. However, while NMDA-iLTP leaved average fluorescence intensity of gephyrin synaptic clusters unchanged, the MMP3-iLTP decreased it. Additionally, exogenous rMMP3 application in $\mathrm{Mmp}^{-/-}$neurons rescued the increase in mIPSC amplitude after iLTP (Fig. 7). These observations provide evidence that MMP3-iLTP at the molecular level belong to the same molecular pathway that underlies the induction of NMDA-iLTP.

Although, to the best of our knowledge, this is the first report demonstrating a key role for MMP3 in GABAergic synaptic plasticity, this enzyme has been previously implicated in glutamatergic synaptic plasticity and related cognitive tasks [22-24]. The involvement of MMP3 in plasticity phenomena is unsurprising because this enzyme is expressed in an activity-dependent manner in the developing and mature brain, where it operates mainly in the extracellular space in the vicinity of synapses [20,36]. Importantly, for glutamatergic synapses, the activity-dependent release and short-term activation of extracellular proteases allow the precise cleavage of adhesion proteins and extracellular matrix (ECM) proteoglycans that, in turn, modify the local milieu of excitatory synapses [11]. However, discernment of the molecular mechanisms whereby MMP3 regulates synaptic signaling is complicated by the rich repertoire of putative MMP substrates [36]. Several potential substrates for MMP3 can be recruited among proteins and glycosaminoglycans of the synaptic adhesive apparatus and brain ECM that are involved in regulating synaptic structure and function [37]. MMP3 can cleave brain proteoglycans, collagens, laminin, tenascins, and extracellular signaling proteins (e.g., pro-brain-derived neurotrophic factor, plasminogen, and cytokines) [36]. Notably, Conant et al. [38] reported that glutamatergic LTP was associated with the MMP3-dependent shedding of intercellular adhesion molecule-5. Whether the cleavage of these substrates is relevant to GABAergic plasticity remains to be elucidated.

In general, the ECM has at least two macroscopic forms: (i) condensed perineuronal nets (PNNs) that enwrap the soma of parvalbumin-positive interneurons and a subset of pyramidal cells and (ii) a diffuse perisynaptic matrix that constitutes $>90 \%$ of the brain ECM [39]. Both forms of ECM, together with the synaptic adhesive apparatus, was proposed to stabilize synaptic structures, and ECM modification through proteolysis may make synapses susceptible to plastic changes and engram encoding [40]. The presence of PNNs limits neuroplasticity, and the disruption of these structures may at least partially relieve this constraint. For example, Pizzorusso et al. [41] reported that the hydrolysis of chondroitin sulfate proteoglycans reactivated ocular dominance plasticity in the adult visual cortex. Similarly, intact PNNs in the amygdala protect fear memories from erasure, because the maturation of perineuronal nets underlie the molecular mechanism that closes a postnatal critical period during which traumatic memories can be erased through an extinction mechanism [42]. However, chondroitinase ABC usually used to hydrolyze PNNs is exogenous and has no endogenous counterpart in the mammalian brain. Future studies should determine whether MMP3 could be an endogenous factor involved in shaping PNNs during GABAergic plasticity and thereby affecting memory formation. This possibility is particularly interesting because several PNN constituents are substrates for MMP3 [36]. Finally, the most prominent PNNs are present on parvalbumin-positive GABAergic interneurons but it is unknown whether these structures affect GABAergic synaptic plasticity.

Several lines of evidence indicate that MMP3 favors iLTP in the present model. There are numerous ways in which the activity of extracellular proteases translates into alterations of the diffusion and synaptic trapping of neurotransmitter receptors. At excitatory synapses, hydrolysis of the brain ECM by hyaluronidase or chondroitinase $\mathrm{ABC}$ was proposed to remove "hurdles" that restrict the lateral diffusion of AMPA receptors, thus allowing the efficient exchange of desensitized receptors to naive ones [43]. Similarly, MMP9, through the cleavage of an unknown substrate, activates integrin signaling that, in turn, increases NMDA receptor mobility in both synaptic and extrasynaptic membranes [44]. Intriguingly, an opposite scenario for $\mathrm{GABA}_{\mathrm{A}}$ receptors was observed in the present study, in which the administration of active MMP3 slowed the diffusion of only synaptic receptors and favored their trapping. Most likely, in the case of iLTP, 
we are dealing with a more complex signaling which does not rely merely on affecting diffusion by altering the physical environment of $\mathrm{GABA}_{\mathrm{A}}$ receptors. However, the exact molecular mechanism of the plasticity that was observed herein remains to be determined.

A precise balance between inhibition and excitation in neuronal networks must be assured by the fine-tuning of inhibitory connections $[4,45]$, which can be achieved by various forms of plasticity that occur at different inhibitory synapses. The present results suggest that the lack of MMP3 activity, which results in impairments in iLTP, enhances hippocampus-dependent spatial learning. This possibility is unsurprising because both proteolysis and the modulation of GABAergic drive are known to affect various forms of learning and memory formation. For example, the enzymatic degradation of glycosaminoglycans by chondroitinase $\mathrm{ABC}$ prolonged object recognition memory [46] and the digestion of brain hyaluronan enhanced cognitive flexibility in gerbils [47]. However, MMP3 deficiency reduces ECM digestion; therefore, the cognitive enhancement that was observed in the present study cannot be ascribed to extensive ECM cleavage. Although anatomical studies ruled out gross morphological changes in CA1 pyramidal neurons of $\mathrm{Mmp3}^{-/-}$mice [48], as well as an impairment in NMDAdependent excitatory LTP [20], it cannot be excluded at the present stage that compensatory changes in the genetically modified animals could have contributed to the observed behavioral phenotype in this model.

Various factors that alter GABAergic inhibition are known to impinge cognition. For example, the genetic knockout of $\alpha 4$ or $\alpha 5$ subunit of $\mathrm{GABA}_{\mathrm{A}}$ receptors improved spatial learning in the Morris water maze and contextual fear conditioning [49, 50]. Similarly, a pharmacological decrease in GABAergic transmission facilitated the acquisition of fear memory and memory retention in passive avoidance learning [51, 52]. Furthermore, the chemogenetic silencing of somatostatin-expressing interneurons during learning was reported to increase the number of neurons that were recruited to the engram and enhanced contextual fear conditioning [53]. In the present study, Mmp3 knockout prevented iLTP, which might also be regarded as a factor debilitating the inhibition. The augmentation of fear conditioning in $\mathrm{Mmp}^{3^{-1}}$ mice may thus be explained by the lack of GABAergic iLTP, but the direct causal link between GABAergic plasticity and alterations of learning requires further studies. In addition, several recent studies have shown that learning is accompanied by plastic changes at GABAergic synapses that constrain memory recall [54]. The lack of such plasticity may be expected to increase memory recall, as observed in $M m p 3^{-1-}$ mice. Nevertheless, while MMP3 is known to contribute to hippocampal-dependent learning, at the present stage, our findings concerning GABAergic plasticity and memory should be regarded as correlative at best, while causative mechanisms still need to be identified.

In conclusion, we provide the first evidence that iLTP strongly relies on MMP3 activity beginning from the early phases of plasticity induction. The present findings also suggest that impairments in NMDA-induced iLTP during learning may enhance memory, but a direct causal link between GABAergic plasticity and learning awaits further investigation.

\section{Materials and methods}

\section{Animals}

The animals of either sex were housed on a natural light/dark (12 h/12 h) cycle and received food and water ad libitum. All of the experiments were performed in accordance with the guidelines of the European Communities Council and approved by the Local Bioethical Committee for Experiments on Laboratory Animals.

\section{Primary hippocampal cultures}

Primary hippocampal cell cultures were prepared from postnatal day $0(\mathrm{P} 0)$ to $\mathrm{P} 2 \mathrm{C} 57 \mathrm{BL} / 6 \mathrm{~J}$ wild-type and $\mathrm{Mmp}^{-/-}$mouse pups [55]. Neurons were plated on polyL-lysine (for single-particle tracking, Sigma-Aldrich) or laminin (Roche) and poly-L-lysine-coated $18 \mathrm{~mm}$-diameter coverslips at a density $2.0 \times 10^{4}$ cells $/ \mathrm{cm}^{2}$ for immunolabeling and $3.1 \times 10^{4}$ cells $/ \mathrm{cm}^{2}$ for electrophysiological recordings. Neuronal cultures were kept in Neurobasal-A medium (Gibco) supplemented with B-27 (1:100; Gibco) at $37{ }^{\circ} \mathrm{C}$ in $5 \% \mathrm{CO}_{2}$. On 2-3 days in vitro (DIV), half of the medium was exchanged for Neurobasal-A/B-27 with Ara-C ( $25 \mu \mathrm{M}$, Sigma-Aldrich). The experiments were performed on cells that were cultured for 12-19 days.

\section{Slice preparation}

Hippocampal slices $(350 \mu \mathrm{m})$ were prepared from 18 - to 21-day-old C57BL/6 wild-type or $\mathrm{Mmp}^{{ }^{-/-}}$mice. After decapitation, the brains were immersed in cold artificial cerebrospinal fluid (aCSF; $119 \mathrm{mM} \mathrm{NaCl}, 26.3 \mathrm{mM} \mathrm{NaHCO}_{3}$, $11 \mathrm{mM}$ glucose, $2.5 \mathrm{mM} \mathrm{KCl}, 1 \mathrm{mM} \mathrm{NaH} \mathrm{PO}_{4}, 1.3 \mathrm{mM}$ $\mathrm{MgSO}_{4}$, and $2.5 \mathrm{mM} \mathrm{CaCl}_{2}, \mathrm{pH}$ 7.4) that was saturated with carbogen $\left(95 \% \mathrm{O}_{2}, 5 \% \mathrm{CO}_{2}\right)$ and cut using a vibrating microtome (Leica VT1200S). After sectioning, the slices were transferred to a recovery chamber that contained the same aCSF at room temperature. 


\section{Electrophysiological recordings}

All of the electrophysiological recordings were performed in aCSF, using borosilicate patch pipettes that were filled with an intracellular solution that contained the following: $10 \mathrm{mM}$ potassium gluconate, $125 \mathrm{mM} \mathrm{KCl}, 1 \mathrm{mM}$ EGTA, $10 \mathrm{mM}$ HEPES, $4 \mathrm{mM} \mathrm{MgATP}$, and $5 \mathrm{mM}$ sucrose, $\mathrm{pH}$ 7.25, 295 MOsm [25]. In slices, after at least a 1-h recovery period, we performed whole-cell patch-clamp recordings of GABA-mediated postsynaptic mIPSCs from hippocampal CA1 pyramidal neurons. Pyramidal cells were first identified visually under a high-power water immersion objective (40X magnification) with infrared differential interference contrast. In the slices, pyramidal cells were also distinguished from interneurons based on their firing pattern consisting of regular train of action potentials and prominent sag occurrence. Baseline mIPSC measurements were recorded for at least $20 \mathrm{~min}$ in the presence of selective blockers of nonNMDA glutamate receptors ( $20 \mu \mathrm{M}$ DNQX) and $\mathrm{Na}^{+}$channels $(1 \mu \mathrm{M}$ tetrodotoxin) at a holding potential of $-70 \mathrm{mV}$. The stability of recordings was checked by monitoring the input resistance during the whole experiment. Cells exhibiting more than $20 \%$ changes were excluded from the analysis. Series resistance was not compensated. Currents were digitized at $20 \mathrm{kHz}$ and filtered at $10 \mathrm{kHz}$ using the MultiClamp 700B amplifier and Axon Digidata 1550 (Molecular Devices). mIPSCs were analyzed manually using pClamp10 software (Molecular Devices).

After stable baseline recordings, we induced inhibitory long-term potentiation (iLTP) by transient exposure to NMDA ( $3 \mathrm{~min}$ in slices, $2 \mathrm{~min} 15 \mathrm{~s}$ in cultures, $20 \mu \mathrm{M}$ ) [25] in wild-type and $\mathrm{Mmp3}^{-/-}$mice. The agonist was then washed out, and mIPSCs were monitored for at least $30 \mathrm{~min}$. The data were binned into 2-min time bins and then averaged to mean amplitude or frequency values at a given time point. The extent of iLTP was defined as the ratio of the mean amplitude of mIPSC recorded 20-22 min after NMDA application to the amplitude recorded before plasticity induction. The mIPSC rise phase was estimated at $10 \%$ to $90 \%$ rise time, and the decay phase was fitted with a biexponential function:

$y(t)=A_{1} \exp \left(-\frac{t}{\tau_{\text {fast }}}\right)+A_{2} \exp \left(-\frac{t}{\tau_{\text {slow }}}\right)$,

where $\tau_{\text {fast }}$ and $\tau_{\text {slow }}$ are the time constants, and $A_{1}$ and $A_{2}$ are the amplitudes of the fast and slow function components, respectively. The mean decay time constant $\left(\tau_{\text {mean }}\right)$ was calculated as $\tau_{\text {mean }}=a_{1} \tau_{\text {fast }}+a_{2} \tau_{\text {slow }}$, where $a_{1}=A 1 /(A 1+A 2)$ and $a_{2}=A 2 /(A 1+A 2)$.

\section{Immunofluorescence staining}

Cells were fixed in a methanol:acetone (1:1) solution for $20 \mathrm{~min}$ at $-20{ }^{\circ} \mathrm{C}$ and then rinsed three times with phosphate-buffered saline (PBS; pH 7.4). Immunolabeling with primary antibodies was preceded by membrane permeabilization $(0.2 \%$ Triton $\mathrm{X}-100$ for $12 \mathrm{~min})$ and incubation with $5 \%$ bovine serum albumin (BSA; Santa Cruz Biotechnology) in PBS for $30 \mathrm{~min}$. Reactions with primary antibodies with $1 \%$ BSA in PBS were run for 90 min at room temperature. Following three washes (1\% BSA in PBS), secondary antibodies with $1 \%$ BSA in PBS were applied for $60 \mathrm{~min}$ at room temperature. After incubation with primary and secondary antibodies, the cells were washed with $1 \%$ BSA in PBS and mounted with Fluoroshield (Sigma-Aldrich).

\section{Confocal imaging and immunofluorescence analysis}

Cells were visualized using an Olympus Fluoview 1000S laser scanning confocal microscope (Olympus, Japan). Images were acquired in the sequential mode as a z-stack with three sections separated by $0.5 \mu \mathrm{m}$ using a $60 \times$ oil immersion objective (PlanApo 1.35) with 473- and 635-nm excitation lasers. On each coverslip, two images from different neurons were analyzed, and the data were averaged. The full analysis of confocal images was performed using ImageJ software (National Institutes of Health and LOCI, University of Wisconsin). Gephyrin clusters were defined as synaptic if they were located within the area of vesicular GABA transporter (vGAT)-positive clusters enlarged by 2 pixels in every direction.

\section{Single-particle tracking imaging of quantum dot-tagged GABAA receptors}

Before quantum dot (QD) labeling, neuronal cell cultures were incubated with anti-vGAT-Oyster550 antibody (Synaptic System) diluted in culture medium at $37^{\circ} \mathrm{C}$ for $20 \mathrm{~min}$ to visualize GABAergic synapses. Antibody specificity was validated previously [30]. Before QD labeling, rabbit antibody directed against an extracellular epitope of $\alpha 1$-subunit of the $\mathrm{GABA}_{\mathrm{A}}$ receptor (AGA-001; Alomone) was incubated for 30 min with anti-rabbit QD 655 (Invitrogen) in the presence of casein (Vectorlab). The neurons were then incubated with the diluted antibody-QD complex for $2 \mathrm{~min}$ at room temperature. The concentration of the antibody-QD complex was adjusted to elicit the staining of $\sim 30$ receptors in the field of view to observe trajectories that in majority are not overlapping. Live-cell imaging was performed at $20 \mathrm{~Hz}$ using a wide-field inverted microscope (Eclipse Ti, Nikon) with a $60 \times$ oil objective (NA 1.4), diode-based illumination (Lumencor, SpectraX Light Engine, Optoprim), an 
EM-CCD camera (9100, Hamamatsu), and Metamorph 7.8 software (Molecular Devices). Band-pass excitation filters $(543 / 22 ; 435 / 40)$ and emission filters $(593 / 40 ; 655 / 15)$ were used to image Oyster550 and QD-655, respectively. Imaging was performed at $32{ }^{\circ} \mathrm{C}$ in a chamber that was continuously perfused with recording solution $(145 \mathrm{mM} \mathrm{NaCl}, 2 \mathrm{mM}$ $\mathrm{KCl}, 2 \mathrm{mM} \mathrm{CaCl}{ }_{2}, 2 \mathrm{mM} \mathrm{MgCl} 2,10 \mathrm{mM}$ glucose, $10 \mu \mathrm{M}$ D-serine, and $10 \mathrm{mM}$ HEPES, $\mathrm{pH}$ 7.4).

The spatial coordinates of QD were determined using MIA software based on simulated annealing algorithm [56]. For a single QD, the coordinates of successive frames were track-connected using custom MATLAB software, which allowed for of displacement of not more than 4 pixels during the maximal allowable dark period between QD blinks (25 frames). If trajectories of two nearby QDs crossed, then both were rejected from further analysis. The mean square displacement (MSD) for a single trajectory was calculated using the following formula:

$\operatorname{MSD}(n \mathrm{~d} t)=(N-n)^{-1} \Sigma_{i=1}^{N-n}\left[\left(x_{i+n}-x_{i}\right)^{2}+\left(y_{i+n}-y_{i}\right)^{2}\right]$,

where $x_{i}$ and $y_{i}$ are the spatial coordinates of a single QD in frame $i, N$ is the total number of points in the trajectory, and $\mathrm{d} t$ is the time interval between successive frames $(50 \mathrm{~ms})$. From the $\operatorname{MSD}(t)$ plot, the instantaneous diffusion coefficient, $D$, was calculated as the slope of the linear function $\operatorname{MSD}(t)=4 \mathrm{Dt}$ fitted to the first 2-4 points. During analysis, QD trajectories were considered synaptic when they colocalized with vGAT-positive GABAergic terminals enlarged by 2 pixels in every direction. The immobile fraction parameter was defined as the relative duration of the residence of a receptor-QD complex in a given compartment with coefficient $<0.0075 \mu \mathrm{m}^{2} \mathrm{~s}^{-1}$. This value has been empirically identified as the minimum of the bimodal distribution of $\log (D)$ function, which reveals the existence of two receptor-QD populations, one mobile and one poorly mobile (immobile). The residence time was calculated as a ratio of the total time spent by QDs at the synapse to the imaging time $(1 \mathrm{~min})$. The MSD vs. time curves were compared using steady-state values and Mann-Whitney $U$ test.

\section{Infusion of NMDA and rMMP3}

In pyramidal neurons, moderate postsynaptic $\mathrm{Ca}^{2+}$ influx through NMDA receptors usually drives iLTP [30], whereas stronger and longer NMDA receptor activation results in the induction of iLTD [33]. The occurrence of GABAergic plasticity is thus determined by the duration of NMDA application and the solution exchange time. All of our electrophysiological recordings were performed in a submerged chamber on an upright microscope, with continuous perfusion with the recording solution at $2.5 \mathrm{ml} /$ min. Under these conditions, we found that iLTP was effectively induced in neuronal cultures by NMDA application (using perfusion pump) for 2 min $15 \mathrm{~s}$. However, in hippocampal slices, the duration of NMDA application needed to be extended to $3 \mathrm{~min}$ (Table 1). This difference [25] may be attributable to a longer solution exchange time in slices compared with cell cultures.

In the experiments with exogenous rMMP3, we applied recombinant protein for different times, depending on the experimental setup. During single-molecule tracking that was performed on an inverted microscope, we acutely treated neuronal cultures with active rMMP3 for $2 \mathrm{~min}$ and exchanged the solution swiftly by pipetting. During electrophysiological recordings of neuronal cultures, we applied rMMP3 through the perfusion pump for $3 \mathrm{~min}$ because shorter times gave inconsistent results that were likely attributable to constrained solution exchange that was caused by the presence of an upright microscope objective in the recording chamber. Additionally, during the gephyrin immunolabeling experiments, we incubated neuronal cultures with rMMP3 for 2 min $15 \mathrm{~s}$ to match the duration of iLTP inducing application of NMDA.

\section{Morris water maze}

Spatial learning ability was tested in a typical hidden-platform Morris water maze (circular pool, $150 \mathrm{~cm}$ diameter) that was filled with $25-26{ }^{\circ} \mathrm{C}$ water that was made opaque with nontoxic white paint as previously described [57, 58]. The mice were trained to find the platform during two blocks of five consecutive daily acquisition sessions, followed by 2 days of rest. Each session consisted of four swimming trials, with a 15-min intertrial interval. Each trial began at one of four starting locations that were randomly selected. The mice were guided to the platform if they failed to find it within $2 \mathrm{~min}$ and remained on the platform for $15 \mathrm{~s}$. The platform remained at a fixed position throughout the learning trials but was removed from the pool during the probe trials. To evaluate retention memory, probe trials were performed on days 8 and 15, during which the mice were allowed to swim freely for $100 \mathrm{~s}$. Swimming paths were recorded using Ethovision equipment and software (Noldus).

\section{Contextual/cued fear conditioning}

The contextual fear conditioning experiment was performed as previously described $[58,59]$. On day 1 , the mice were placed in the testing chamber (dark Plexiglas cage with a grid floor) and allowed to habituate to it for $5 \mathrm{~min}$. On day 2 , the mice were placed in the testing chamber. After a 2-min baseline exploration period, a tone cue $(4 \mathrm{kHz}, 80 \mathrm{~dB})$ that served as the conditioned stimulus (CS) was delivered for $30 \mathrm{~s}$, which co-terminated with a 2-s mild footshock 
Table 1 Duration of treatments used to induce NMDA-iLTP and MMP3-iLTP in different experimental conditions

\begin{tabular}{lllll}
\hline Substance application & $\begin{array}{l}\text { Brain slices electrophysiol- } \\
\text { ogy (upright microscope) }\end{array}$ & $\begin{array}{l}\text { Neuronal culture elec- } \\
\text { trophysiology (upright } \\
\text { microscope) }\end{array}$ & $\begin{array}{l}\text { Neuronal culture immunola- } \\
\text { beling (12-well plate) }\end{array}$ & $\begin{array}{l}\text { Neuronal culture SMT } \\
\text { (inverted microscope) }\end{array}$ \\
\hline $\begin{array}{l}\text { Application of NMDA to } \\
\text { induce NMDA-iLTP }\end{array}$ & 3 min (Fig. 1,2,3) & $\begin{array}{l}2 \text { min 15 s (Fig. 4a-c; } \\
7 \mathrm{a}, \mathrm{c}-\mathrm{d})\end{array}$ & 2 min 15 s (Fig. 4d-g) & 2 min (Fig. 5) \\
$\begin{array}{l}\text { Application of rMMP3 to } \\
\text { induce MMP3-iLTP }\end{array}$ & - & $3 \min$ (Fig. 6a-d) & 2 min 15 s (Fig. 6i, j) & 2 min (Fig. 6e-h) \\
$\begin{array}{l}\text { Application of NMDA with } \\
\text { rMMP3 }\end{array}$ & - & 2 min 15 s (Fig. 7b-d) & 2 min 15 s (Fig. 6k, l) & - \\
$\begin{array}{l}\text { Prolonged application of } \\
\text { NMDA }\end{array}$ & - & 2 min 45 s (Suppl Fig. 8a-c) & 2 min 45 s (Suppl Fig. 8d-f) & - \\
\hline
\end{tabular}

(0.3 $\mathrm{mA})$ that served as the unconditioned stimulus (US). After $60 \mathrm{~s}$, this CS-US pairing was repeated, followed by $30 \mathrm{~s}$ of exploration. On day 3 , memory retention of either the context or tone was assessed. At first, the mice were placed in the cage for $5 \mathrm{~min}$ without CS or US presentation (context test). After 2-3 h, the mice were placed in a new context (no grid floor, lights on, and mint odor) for $3 \mathrm{~min}$ (pre-CS period), and then a 3-min tone was presented without the US (CS test period). The percent time spent freezing was recorded and analyzed for each time period in the various experimental sessions. See Fig. 8e for a schematic overview.

\section{Drugs and antibodies}

We used the following MMP inhibitors: FN-439 $(180 \mu \mathrm{M}$, Calbiochem), UK-356618 (2 $\mu \mathrm{M}$, Sigma-Aldrich), and SB3CT (10 $\mu \mathrm{M}$, Sigma-Aldrich). FN-439 is a broad-spectrum MMP inhibitor that inhibits MMP1, MMP2, MMP3, MMP8, and MMP9 at the tested concentration. UK-356618 inhibits the activity of MMP3 and MMP13 at $2 \mu \mathrm{M}$ and partially blocks MMP9 $\left(K_{i}\right.$ for MMP9 $\left.=840 \mathrm{nM}\right)$. SB-3CT is a specific inhibitor of MMP2 and MMP9 $\left(K_{i}\right.$ for MMP2 $=14 \mathrm{nM}$; $K_{i}$ for MMP9 $\left.=600 \mathrm{nM}\right)$ [20]. Recombinant human active rMMP3 (Sigma, catalog no. SRP7783) was applied at a concentration of $400 \mathrm{ng} / \mathrm{ml}$.

Anti-gephyrin (catalog no. 147 111, 1:500) and antivGAT (catalog no. 131 006, 1:500) antibodies were purchased from Synaptic System. Alexa 488 and Alexa 633 fluorescent secondary antibodies against mouse and chicken antibodies were obtained from ThermoFisher Scientific.

\section{Statistical analysis}

The statistical analyses of data were performed using SigmaPlot (Systat Software) and GraphPad Prism 8. The electrophysiological and immunocytochemical data are expressed as mean \pm SEM; the diffusion coefficients measured using single-particle tracking approach are expressed as median \pm interquartile range (IQR 25-75\%). Datasets with a normal distribution were compared using two-tailed Student's $t$ test (paired or unpaired), one-way or two-way analysis of variance (ANOVA) with Bonferroni correction. Datasets with a non-Gaussian distribution were analyzed using the nonparametric two-tailed Mann-Whitney $U$ test (unpaired comparisons) or Wilcoxon signed-rank test (paired comparisons). All statistical tests were two-tailed and were specified in figure legends. The sample sizes are specified in the graphs. For the cell culture electrophysiological and morphological experiments, $n$ refers to the number of coverslips with neurons from at least three different preparations. Values of $p<0.05$ were considered statistically significant.

Acknowledgments We are grateful to Anna Buszka for experimental support.

Author contributions JWM, GW designed and supervised the study; $\mathrm{KL}, \mathrm{PB}, \mathrm{GW}$, AML, performed electrophysiological recordings and their analysis; AML performed immunocytochemical studies; GW, EMP, AB performed single-particle tracking studies; IVH, LDG, LM performed behavioral studies; GW, JWM, KL, AML, IVH, wrote the manuscript with critical input from all authors.

Funding The project was supported by National Science Centre (Poland) grant SONATA 2017/26/D/NZ4/00450 (to GW). LDG is supported by the Research Foundation-Flanders (Belgium). PB is supported by the Foundation for Polish Science (FNP) 2019/2020 and by Polish National Science Centre scholarship ETIUDA 2018/28/T/ NZ4/00344. JWM was supported by the National Science Center (NCN) grant UMO-2018/31/B/NZ4/01998.

\section{Compliance with ethical standards}

Conflict of interest The authors declare that they have no conflict of interest.

Ethics approval All of the experiments were performed in accordance with the guidelines of the European Communities Council and approved by the Local Bioethical Committee for Experiments on Laboratory Animals.

Open Access This article is licensed under a Creative Commons Attribution 4.0 International License, which permits use, sharing, 
adaptation, distribution and reproduction in any medium or format, as long as you give appropriate credit to the original author(s) and the source, provide a link to the Creative Commons licence, and indicate if changes were made. The images or other third party material in this article are included in the article's Creative Commons licence, unless indicated otherwise in a credit line to the material. If material is not included in the article's Creative Commons licence and your intended use is not permitted by statutory regulation or exceeds the permitted use, you will need to obtain permission directly from the copyright holder. To view a copy of this licence, visit http://creativecommons .org/licenses/by/4.0/.

\section{References}

1. Josselyn SA, Kohler S, Frankland PW (2015) Finding the engram. Nature reviews. Neuroscience 16(9):521-534. https:// doi.org/10.1038/nrn4000

2. Bliss TV, Lomo T (1973) Long-lasting potentiation of synaptic transmission in the dentate area of the anaesthetized rabbit following stimulation of the perforant path. J Physiol 232(2):331-356

3. Choi JH, Sim SE (2018) Interregional synaptic maps among engram cells underlie memory formation. Science 360(6387):430435. https://doi.org/10.1126/science.aas9204

4. Chiu CQ, Barberis A, Higley MJ (2019) Preserving the balance: diverse forms of long-term GABAergic synaptic plasticity. Nat Rev Neurosci 20(5):272-281. https://doi.org/10.1038/s4158 3-019-0141-5

5. Castillo PE, Chiu CQ, Carroll RC (2011) Long-term plasticity at inhibitory synapses. Curr Opin Neurobiol 21(2):328-338. https ://doi.org/10.1016/j.conb.2011.01.006

6. Chevaleyre V, Castillo PE (2003) Heterosynaptic LTD of hippocampal GABAergic synapses: a novel role of endocannabinoids in regulating excitability. Neuron 38(3):461-472. https:// doi.org/10.1016/s0896-6273(03)00235-6

7. Vickers ED, Clark C, Osypenko D, Fratzl A, Kochubey O, Bettler B, Schneggenburger R (2018) Parvalbumin-interneuron output synapses show spike-timing-dependent plasticity that contributes to auditory map remodeling. Neuron 99(4):720-735.e726. https:// doi.org/10.1016/j.neuron.2018.07.018

8. Vogels TP, Sprekeler H, Zenke F, Clopath C, Gerstner W (2011) Inhibitory plasticity balances excitation and inhibition in sensory pathways and memory networks. Science (New York, NY) 334(6062):1569-1573. https://doi.org/10.1126/science.1211095

9. Hennequin G, Agnes EJ, Vogels TP (2017) Inhibitory plasticity: balance, control, and codependence. Annu Rev Neurosci 40:557579. https://doi.org/10.1146/annurev-neuro-072116-031005

10. Koolschijn RS, Emir UE, Pantelides AC, Nili H, Behrens TEJ, Barron HC (2019) The hippocampus and neocortical inhibitory engrams protect against memory interference. Neuron 101(3):528-541.e526. https://doi.org/10.1016/j.neuro n. 2018.11 .042

11. Nagappan-Chettiar S, Johnson-Venkatesh EM, Umemori H (2017) Activity-dependent proteolytic cleavage of cell adhesion molecules regulates excitatory synaptic development and function. Neurosci Res 116:60-69. https://doi.org/10.1016/j.neure s.2016.12.003

12. Sonderegger P, Matsumoto-Miyai K (2014) Activity-controlled proteolytic cleavage at the synapse. Trends Neurosci (in press). https://doi.org/10.1016/j.tins.2014.05.007

13. Nagy V, Bozdagi O, Matynia A, Balcerzyk M, Okulski P, Dzwonek J, Costa RM, Silva AJ, Kaczmarek L, Huntley GW (2006) Matrix metalloproteinase-9 is required for hippocampal late-phase long-term potentiation and memory. J Neurosci 26(7):1923-1934. https://doi.org/10.1523/jneurosci.4359-05.2006

14. Padamsey Z, McGuinness L, Bardo SJ, Reinhart M, Tong R, Hedegaard A, Hart ML, Emptage NJ (2017) Activity-dependent exocytosis of lysosomes regulates the structural plasticity of dendritic spines. Neuron 93(1):132-146. https://doi.org/10.1016/j. neuron.2016.11.013

15. Bijata M, Labus J, Guseva D, Stawarski M, Butzlaff M, Dzwonek J, Schneeberg J, Bohm K, Michaluk P, Rusakov DA, Dityatev A, Wilczynski G, Wlodarczyk J, Ponimaskin E (2017) Synaptic remodeling depends on signaling between serotonin receptors and the extracellular matrix. Cell Rep 19(9):1767-1782. https://doi. org/10.1016/j.celrep.2017.05.023

16. Stefaniuk M, Beroun A, Lebitko T, Markina O, Leski S, Meyza K, Grzywacz A, Samochowiec J, Samochowiec A, Radwanska K, Kaczmarek L (2017) Matrix metalloproteinase-9 and synaptic plasticity in the central amygdala in control of alcohol-seeking behavior. Biol Psychiat 81(11):907-917. https://doi.org/10.1016/j. biopsych.2016.12.026

17. Smith AC, Kupchik YM (2014) Synaptic plasticity mediating cocaine relapse requires matrix metalloproteinases. Nat Neurosci 17(12):1655-1657. https://doi.org/10.1038/nn.3846

18. van der Kooij MA, Fantin M, Rejmak E, Grosse J, Zanoletti O, Fournier C, Ganguly K, Kalita K, Kaczmarek L, Sandi C (2014) Role for MMP-9 in stress-induced downregulation of nectin-3 in hippocampal CA1 and associated behavioural alterations. Nat Commun 5:4995. https://doi.org/10.1038/ncomms5995

19. Aerts J, Nys J, Moons L, Hu TT, Arckens L (2015) Altered neuronal architecture and plasticity in the visual cortex of adult MMP-3-deficient mice. Brain Struct Funct 220(5):2675-2689. https://doi.org/10.1007/s00429-014-0819-4

20. Wiera G, Nowak D, van Hove I, Dziegiel P, Moons L, Mozrzymas JW (2017) Mechanisms of NMDA receptor- and voltage-gated L-type calcium channel-dependent hippocampal LTP critically rely on proteolysis that is mediated by distinct metalloproteinases. J Neurosci 37(5):1240-1256. https://doi.org/10.1523/jneur osci.2170-16.2016

21. Brzdak P, Wojcicka O, Zareba-Koziol M, Minge D, Henneberger C, Wlodarczyk J, Mozrzymas JW, Wojtowicz T (2019) Synaptic Potentiation at Basal and Apical Dendrites of Hippocampal Pyramidal Neurons Involves Activation of a Distinct Set of Extracellular and Intracellular Molecular Cues. Cerebral cortex (New York, NY : 1991) 29 (1):283-304. 10.1093/cercor/bhx324

22. Wright JW, Harding JW (2009) Contributions of matrix metalloproteinases to neural plasticity, habituation, associative learning and drug addiction. Neural Plasticity 2009:579382. https://doi. org/10.1155/2009/579382

23. Meighan SE, Meighan PC, Choudhury P, Davis CJ, Olson ML, Zornes PA, Wright JW, Harding JW (2006) Effects of extracellular matrix-degrading proteases matrix metalloproteinases 3 and 9 on spatial learning and synaptic plasticity. J Neurochem 96(5):12271241. https://doi.org/10.1111/j.1471-4159.2005.03565.x

24. Olson ML, Meighan PC, Brown TE, Asay AL, Benoist CC, Harding JW, Wright JW (2008) Hippocampal MMP-3 elevation is associated with passive avoidance conditioning. Regul Pept 146(1-3):19-25. https://doi.org/10.1016/j.regpep.2007.07.004

25. Marsden KC, Beattie JB, Friedenthal J, Carroll RC (2007) NMDA receptor activation potentiates inhibitory transmission through GABA receptor-associated protein-dependent exocytosis of GABA(A) receptors. J Neurosci 27(52):14326-14337. https:// doi.org/10.1523/jneurosci.4433-07.2007

26. Chiu CQ, Martenson JS, Yamazaki M, Natsume R, Sakimura K, Tomita S, Tavalin SJ, Higley MJ (2018) Input-specific NMDARdependent potentiation of dendritic GABAergic inhibition. Neuron 97(2):368-377.e363. https://doi.org/10.1016/j.neuro n.2017.12.032 
27. Kano M, Rexhausen U, Dreessen J, Konnerth A (1992) Synaptic excitation produces a long-lasting rebound potentiation of inhibitory synaptic signals in cerebellar Purkinje cells. Nature 356(6370):601-604. https://doi.org/10.1038/356601a0

28. Mudgett JS, Hutchinson NI, Chartrain NA, Forsyth AJ, McDonnell J, Singer II, Bayne EK, Flanagan J, Kawka D, Shen CF, Stevens K, Chen H, Trumbauer M, Visco DM (1998) Susceptibility of stromelysin 1-deficient mice to collagen-induced arthritis and cartilage destruction. Arthritis Rheum 41(1):110-121. https://doi. org/10.1002/1529-0131(199801)41:1<110:aid-art14>3.0.co;2-g

29. Pennacchietti F, Vascon S, Nieus T (2017) Nanoscale molecular reorganization of the inhibitory postsynaptic density is a determinant of GABAergic synaptic potentiation. J Neurosci 37(7):17471756. https://doi.org/10.1523/jneurosci.0514-16.2016

30. Petrini EM, Ravasenga T, Hausrat TJ, Iurilli G, Olcese U, Racine V, Sibarita JB, Jacob TC, Moss SJ, Benfenati F, Medini P, Kneussel M, Barberis A (2014) Synaptic recruitment of gephyrin regulates surface GABAA receptor dynamics for the expression of inhibitory LTP. Nat Commun 5:3921. https://doi.org/10.1038/ ncomms 4921

31. Choquet D, Triller A (2013) The dynamic synapse. Neuron 80(3):691-703. https://doi.org/10.1016/j.neuron.2013.10.013

32. Marsden KC, Shemesh A, Bayer KU, Carroll RC (2010) Selective translocation of $\mathrm{Ca} 2+/$ calmodulin protein kinase IIalpha (CaMKIIalpha) to inhibitory synapses. Proc Natl Acad Sci USA 107(47):20559-20564. https://doi.org/10.1073/pnas.1010346107

33. Bannai H, Niwa F, Sherwood MW, Shrivastava AN, Arizono M, Miyamoto A, Sugiura K, Levi S, Triller A, Mikoshiba K (2015) Bidirectional control of synaptic GABAAR clustering by glutamate and calcium. Cell Rep 13(12):2768-2780. https://doi. org/10.1016/j.celrep.2015.12.002

34. Latif-Hernandez A, Shah D, Ahmed T, Lo AC, Callaerts-Vegh Z, Van der Linden A, Balschun D, D'Hooge R (2016) Quinolinic acid injection in mouse medial prefrontal cortex affects reversal learning abilities, cortical connectivity and hippocampal synaptic plasticity. Sci Rep 6:36489. https://doi.org/10.1038/srep36489

35. Orlando M, Ravasenga T, Petrini EM, Falqui A (2017) Correlating Fluorescence and High-Resolution Scanning Electron Microscopy (HRSEM) for the study of GABAA receptor clustering induced by inhibitory synaptic plasticity. Sci Rep 7(1):13768. https://doi. org/10.1038/s41598-017-14210-5

36. Van Hove I, Lemmens K, Van de Velde S, Verslegers M, Moons L (2012) Matrix metalloproteinase-3 in the central nervous system: a look on the bright side. J Neurochem 123(2):203-216. https:// doi.org/10.1111/j.1471-4159.2012.07900.x

37. Miyata S, Kitagawa H (2017) Formation and remodeling of the brain extracellular matrix in neural plasticity: roles of chondroitin sulfate and hyaluronan. Biochim Biophys Acta Gen Subjects (1861) 10:2420-2434. https://doi.org/10.1016/j.bbage n.2017.06.010

38. Conant K, Wang Y, Szklarczyk A, Dudak A, Mattson MP, Lim ST (2010) Matrix metalloproteinase-dependent shedding of intercellular adhesion molecule-5 occurs with long-term potentiation. Neuroscience 166(2):508-521. https://doi.org/10.1016/j. neuroscience.2009.12.061

39. Fawcett JW, Oohashi T (2019) The roles of perineuronal nets and the perinodal extracellular matrix in neuronal function. Nat Rev Neurosci 20(8):451-465. https://doi.org/10.1038/s4158 3-019-0196-3

40. Tsien RY (2013) Very long-term memories may be stored in the pattern of holes in the perineuronal net. Proc Natl Acad Sci USA 110(30):12456-12461. https://doi.org/10.1073/pnas.13101 58110/-/DCSupplemental

41. Pizzorusso T, Medini P, Berardi N, Chierzi S, Fawcett JW, Maffei L (2002) Reactivation of ocular dominance plasticity in the adult visual cortex. Science (New York, NY) 298(5596):12481251. https://doi.org/10.1126/science.1072699

42. Gogolla N, Caroni P, Luthi A, Herry C (2009) Perineuronal nets protect fear memories from erasure. Science (New York, NY) 325(5945):1258-1261. https://doi.org/10.1126/science.1174146

43. Frischknecht R, Heine M, Perrais D, Seidenbecher CI, Choquet D, Gundelfinger ED (2009) Brain extracellular matrix affects AMPA receptor lateral mobility and short-term synaptic plasticity. Nat Neurosci 12(7):897-904. https://doi.org/10.1038/ nn. 2338

44. Michaluk P, Mikasova L, Groc L, Frischknecht R, Choquet D, Kaczmarek L (2009) Matrix metalloproteinase-9 controls NMDA receptor surface diffusion through integrin beta1 signaling. J Neurosci 29(18):6007-6012. https://doi.org/10.1523/JNEUR OSCI.5346-08.2009

45. Barberis A (2019) Postsynaptic plasticity of GABAergic synapses. Neuropharmacology. https://doi.org/10.1016/j.neuropharm 2019.05.020

46. Romberg C, Yang S, Melani R, Andrews MR, Horner AE, Spillantini MG, Bussey TJ, Fawcett JW, Pizzorusso T, Saksida LM (2013) Depletion of perineuronal nets enhances recognition memory and long-term depression in the perirhinal cortex. J Neurosci 33(16):7057-7065. https://doi.org/10.1523/jneur osci.6267-11.2013

47. Happel MF, Niekisch H, Castiblanco Rivera LL, Ohl FW, Deliano M, Frischknecht R (2014) Enhanced cognitive flexibility in reversal learning induced by removal of the extracellular matrix in auditory cortex. Proc Natl Acad Sci USA 111(7):2800-2805. https://doi.org/10.1073/pnas.1310272111

48. Nowak D, Groef L, Moons L, Mozrzymas JW (2018) MMP-3 deficiency does not influence the length and number of CA1 dendrites of hippocampus of adult mice. Acta Neurobiol Exp 78(3):281-286

49. Moore MD, Cushman J, Chandra D, Homanics GE, Olsen RW, Fanselow MS (2010) Trace and contextual fear conditioning is enhanced in mice lacking the alpha4 subunit of the GABA(A) receptor. Neurobiol Learn Mem 93(3):383-387. https://doi. org/10.1016/j.nlm.2009.12.004

50. Collinson N, Kuenzi FM, Jarolimek W, Maubach KA, Cothliff R, Sur C, Smith A, Otu FM, Howell O, Atack JR, McKernan RM, Seabrook GR, Dawson GR, Whiting PJ, Rosahl TW (2002) Enhanced learning and memory and altered GABAergic synaptic transmission in mice lacking the alpha 5 subunit of the GABAA receptor. J Neurosci 22(13):5572-5580. Doi:20026436

51. Makkar SR, Zhang SQ, Cranney J (2010) Behavioral and neural analysis of GABA in the acquisition, consolidation, reconsolidation, and extinction of fear memory. Neuropsychopharmacology 35(8):1625-1652. https://doi.org/10.1038/npp.2010.53

52. Zarrindast MR, Bakhsha A, Rostami P, Shafaghi B (2002) Effects of intrahippocampal injection of GABAergic drugs on memory retention of passive avoidance learning in rats. J Psychopharmacol (Oxford, England) 16(4):313-319. https://doi.org/10.1177/02698 8110201600405

53. Stefanelli T, Bertollini C, Luscher C, Muller D, Mendez P (2016) Hippocampal somatostatin interneurons control the size of neuronal memory ensembles. Neuron 89(5):1074-1085. https://doi. org/10.1016/j.neuron.2016.01.024

54. Barron HC, Vogels TP, Behrens TE, Ramaswami M (2017) Inhibitory engrams in perception and memory. Proc Natl Acad Sci USA 114(26):6666-6674. https://doi.org/10.1073/pnas.1701812114

55. Kaczor PT, Mozrzymas JW (2017) Key metabolic enzymes underlying astrocytic upregulation of GABAergic plasticity. Front Cell Neurosci 11:144. https://doi.org/10.3389/fncel.2017.00144

56. Racine V, Hertzog A, Jouanneau J, Salamero J, Kervrann C, Sibarita JB (2006) Multiple-target tracking of 3D fluorescent objects based on simulated annealing. In: 3rd IEEE international 
symposium on biomedical imaging conference: nano to macro, $\mathrm{pp}$ 1020-1023. https://doi.org/10.1109/ISBI.2006.1625094

57. Laeremans A, Sabanov V, Ahmed T, Nys J, Van de Plas B, Vinken K, Woolley DG, Gantois I, D’Hooge R, Arckens L, Balschun D (2015) Distinct and simultaneously active plasticity mechanisms in mouse hippocampus during different phases of Morris water maze training. Brain Struct Funct 220(3):1273-1290. https://doi. org/10.1007/s00429-014-0722-z

58. Goddyn H, Leo S, Meert T, D'Hooge R (2006) Differences in behavioural test battery performance between mice with hippocampal and cerebellar lesions. Behav Brain Res 173(1):138147. https://doi.org/10.1016/j.bbr.2006.06.016
59. Nunes AF, Amaral JD, Lo AC, Fonseca MB, Viana RJ, CallaertsVegh Z, D'Hooge R, Rodrigues CM (2012) TUDCA, a bile acid, attenuates amyloid precursor protein processing and amyloid-beta deposition in APP/PS1 mice. Mol Neurobiol 45(3):440-454. https ://doi.org/10.1007/s12035-012-8256-y

Publisher's Note Springer Nature remains neutral with regard to jurisdictional claims in published maps and institutional affiliations. 